Received: 17 October 2011, Accepted: 18 March 2012

Edited by: J. Pullin

Reviewed by: L. Freidel, Perimeter Institute for Theoretical Physics,

www.papersinphysics.org

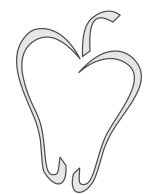

ISSN 1852-4249

\title{
Invited review: The new spin foam models and quantum gravity
}

\author{
Alejandro Perez ${ }^{1 *}$ \\ In this article, we give a systematic definition of the recently introduced spin foam models \\ for four-dimensional quantum gravity, reviewing the main results on their semiclassical \\ limit on fixed discretizations.
}

\section{Introduction}

The quantization of the gravitational interaction is a major open challenge in theoretical physics. This review presents the status of the spin foam approach to the problem. Spin foam models are definitions of the path integral formulation of quantum general relativity and are expected to be the covariant counterpart of the background independent canonical quantization of general relativity known as loop quantum gravity [1-3].

This article focuses on the definition of the recently introduced Engle-Pereira-Rovelli-Livine (EPRL) model $[4,5]$ and the closely related FreidelKrasnov (FK) model [6]. An important original feature of the present paper is the explicit derivation of both the Riemannian and the Lorentzian models, in terms of a notation that exhibits the close relationship between the two, at the algebraic level, that might signal a possible deeper relationship at the level of transition amplitudes.

We will take Plebanski's perspective in which general relativity is formulated as a constrained $\mathrm{BF}$ theory (for a review introducing the new models from a bottom-up perspective see Ref. [7]; for an extended version of the present review including a wide collection of related work see Ref. [8]). For that reason, it will be convenient to start this review by introducing the exact spin foam quantization of BF in the following section. In Section III, we present the EPRL model in both its Riemannian and Lorentzian versions. A unified treatment of the representation theory of the relevant gauge groups is presented in that section. In Section IV, we introduce the FK model and discuss its relationship with the EPRL model. In Section V, we describe the structure of the boundary states of these models and emphasize the relationship with the kinematical Hilbert space of loop quantum gravity. In Section VI, we give a compendium of important issues (and associated references) that have been left out but which are important for future development. Finally, in Section VII, we present the recent encouraging results of the nature of the semiclassical limit of the new models.

*E-mail: perez@cpt.univ-mrs.fr

1 Centre de Physique Théorique, Campus de Luminy, 13288 Marseille, France. Unité Mixte de Recherche (UMR 6207) du CNRS et des Universités Aix-Marseille I, Aix-Marseille II, et du Sud Toulon-Var; laboratoire afilié à la FRUMAM (FR 2291). 
Papers in Physics, vol. 4, ART. 040004 (2012) / A. Perez

\section{Spin foam quantization of $\mathrm{BF}$ theory}

We will start by briefly reviewing the spin foam quantization of BF theory. This section will be the basic building block for the construction of the models of quantum gravity that are dealt with in this article. The key idea is that the quantum transition amplitudes (computed in the path integral representation) of gravity can be obtained by suitably restricting the histories that are summed over in the spin foam representation of exactly solvable BF theory. We describe the nature of these constraints at the end of this section.

Here, one follows the perspective of Ref. [9]. Let $G$ be a compact group whose Lie algebra $\mathfrak{g}$ has an invariant inner product, here denoted \langle\rangle , and $\mathcal{M}$ a d-dimensional manifold. Classical BF theory is defined by the action

$$
S[\mathrm{~B}, \omega]=\int_{\mathcal{M}}\langle\mathrm{B} \wedge \mathrm{F}(\omega)\rangle,
$$

where B is a $\mathfrak{g}$ valued $(\mathrm{d}-2)$-form, $\omega$ is a connection on a $G$ principal bundle over $\mathcal{M}$. The theory has no local excitations: All the solutions of the equations of motion are locally related by gauge transformations. More precisely, the gauge symmetries of the action are the local $G$ gauge transformations

$$
\delta \mathrm{B}=[\mathrm{B}, \alpha], \quad \delta \omega=\mathrm{d}_{\omega} \alpha,
$$

where $\alpha$ is a $\mathfrak{g}$-valued 0 -form, and the 'topological' gauge transformation

$$
\delta \mathrm{B}=\mathrm{d}_{\omega} \eta, \quad \delta \omega=0,
$$

where $d_{\omega}$ denotes the covariant exterior derivative and $\eta$ is a $\mathfrak{g}$-valued 0 -form. The first invariance is manifest in the form of the action, while the second one is a consequence of the Bianchi identity, $\mathrm{d}_{\omega} F(\omega)=0$. The gauge symmetries are so vast that all the solutions to the equations of motion are locally pure gauge. The theory has only global or topological degrees of freedom.

For the time being, we assume $\mathcal{M}$ to be a compact and orientable manifold. The partition function, $\mathcal{Z}$, is formally given by

$$
\mathcal{Z}=\int \mathcal{D}[\mathrm{B}] \mathcal{D}[\omega] \quad \exp \left(i \int_{\mathcal{M}}\langle\mathrm{B} \wedge F(\omega)\rangle\right) .
$$

Formally integrating over the B field in (4), we obtain

$$
\mathcal{Z}=\int \mathcal{D}[\omega] \delta(F(\omega))
$$

The partition function $\mathcal{Z}$ corresponds to the 'volume' of the space of flat connections on $\mathcal{M}$.

In order to give a meaning to the formal expressions above, we replace the d-dimensional manifold $\mathcal{M}$ with an arbitrary cellular decomposition $\Delta$. We also need the notion of the associated dual 2complex of $\Delta$ denoted by $\Delta^{\star}$. The dual 2-complex $\Delta^{\star}$ is a combinatorial object defined by a set of vertices $v \in \Delta^{\star}$ (dual to d-cells in $\Delta$ ) edges $e \in \Delta^{\star}$ (dual to $(\mathrm{d}-1)$-cells in $\Delta$ ) and faces $f \in \Delta^{\star}$ (dual to $(\mathrm{d}-2)$-cells in $\Delta)$. In the case where $\Delta$ is a simplicial decomposition of $\mathcal{M}$, the structure of both $\Delta$ and $\Delta^{\star}$ is illustrated in Figs. 1, 2 and 3 in two, three, and four dimensions, respectively.
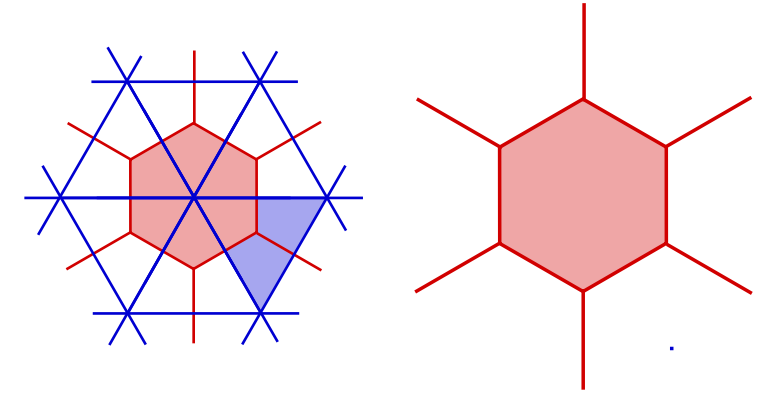

Figure 1: On the left: A triangulation and its dual in two dimensions. On the right: The dual two complex; faces (shaded polygon) are dual to 0 -simplices in $2 \mathrm{~d}$.
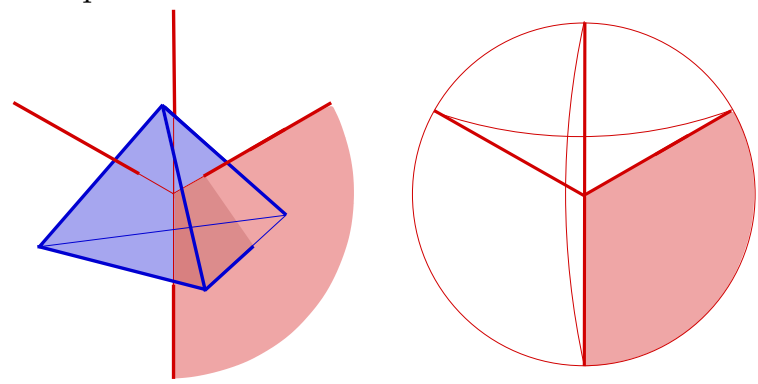

Figure 2: On the left: A triangulation and its dual in three dimensions. On the right: The dual two complex; faces (shaded wedge) are dual to 1simplices in $3 \mathrm{~d}$. 

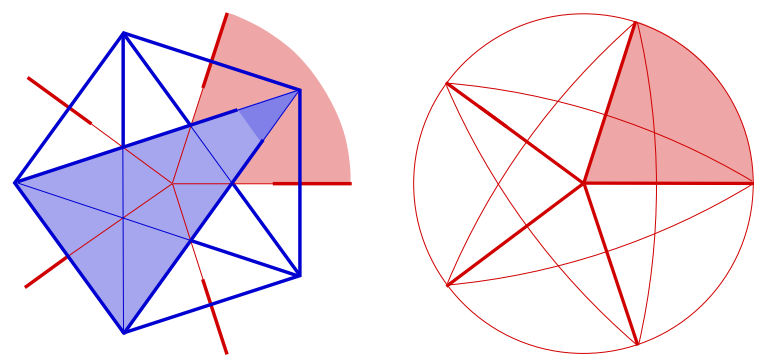

Figure 3: On the left: a triangulation and its dual in four dimensions. On the right: the dual two complex; faces (shaded wedge) are dual to triangles in $4 \mathrm{~d}$. The shaded triangle dual to the shaded face is exhibited.

For simplicity, we concentrate on the case when $\Delta$ is a triangulation. The field $\mathrm{B}$ is associated with Lie algebra elements $B_{f}$ assigned to faces $f \in \Delta^{\star}$. We can think of it as the integral of the $(\mathrm{d}-2)$-form B on the (d-2)-cell dual to the face $f \in \Delta^{\star}$, namely

$$
B_{f}=\int_{(\mathrm{d}-2)-\text { cell }} \mathrm{B} .
$$

In other words, $B_{f}$ can be interpreted as the 'smearing' of the continuous (d-2)-form B on the (d-2)-cells in $\Delta$. We use the one-to-one correspondence between faces $f \in \Delta^{\star}$ and (d-2)-cells in $\Delta$ to label the discretization of the $\mathrm{B}$ field $B_{f}$. The connection $\omega$ is discretized by the assignment of group elements $g_{e} \in G$ to edges $e \in \Delta^{\star}$. One can think of the group elements $g_{e}$ as the holonomy of $\omega$ along $e \in \Delta^{\star}$, namely

$$
g_{e}=\mathrm{P} \exp \left(-\int_{e} \omega\right),
$$

where the symbol "P exp" denotes the path-orderexponential that reminds us of the relationship of the holonomy with the connection along the path $e \in \Delta^{\star}$.

With this, the discretized version of the path integral (4) is

$$
\begin{aligned}
Z(\Delta) & =\int \prod_{e \in \Delta^{\star}} d g_{e} \prod_{f \in \Delta^{\star}} d B_{f} e^{i B_{f} U_{f}} \\
& =\int \prod_{e \in \Delta^{\star}} d g_{e} \prod_{f \in \Delta^{\star}} \delta\left(g_{e_{1}} \cdots g_{e_{n}}\right),
\end{aligned}
$$

where $U_{f}=g_{e_{1}} \cdots g_{e_{n}}$ denotes the holonomy around faces, and the second equation is the result of the B integration: It can be, thus, regarded as the analog of (5). The integration measure $d B_{f}$ is the standard Lebesgue measure, while the integration in the group variables is done in terms of the invariant measure in $G$ (which is the unique Haar measure when $G$ is compact). For given $h \in G$ and test function $F(g)$, the invariance property reads as follows

$$
\begin{aligned}
\int d g F(g)=\int d g F\left(g^{-1}\right) & =\int d g F(g h) \\
& =\int d g F(h g)
\end{aligned}
$$

The Peter-Weyl's theorem provides a useful formula or the Dirac delta distribution appearing in (8), namely

$$
\delta(g)=\sum_{\rho} d_{\rho} \operatorname{Tr}[\rho(g)],
$$

where $\rho$ are irreducible unitary representations of $G$. From the previous expression, one obtains

$$
\begin{aligned}
\mathcal{Z}(\Delta) & =\sum_{\mathcal{C}:\{\rho\} \rightarrow\{f\}} \\
& \int \prod_{e \in \Delta^{\star}} d g_{e} \prod_{f \in \Delta^{\star}} \mathrm{d}_{\rho_{f}} \operatorname{Tr}\left[\rho_{f}\left(g_{e}^{1} \ldots g_{e}^{N}\right)\right]
\end{aligned}
$$

Integration over the connection can be performed as follows. In a triangulation $\Delta$, the edges $e \in \Delta^{\star}$ bound precisely $\mathrm{d}$ different faces. Therefore, the $g_{e}$ 's in (11) appear in d different traces. The relevant formula is

$$
\begin{aligned}
& P_{i n v}^{e}\left(\rho_{1}, \cdots, \rho_{\mathrm{d}}\right) \\
& :=\int d g_{e} \rho_{1}\left(g_{e}\right) \otimes \rho_{2}\left(g_{e}\right) \otimes \cdots \otimes \rho_{\mathrm{d}}\left(g_{e}\right) .
\end{aligned}
$$

For compact $G$, using the invariance (and normalization) of the the integration measure (9), it is easy to prove that $P_{i n v}^{e}=\left(P_{i n v}^{e}\right)^{2}$ is the projector onto $\operatorname{Inv}\left[\rho_{1} \otimes \rho_{2} \otimes \cdots \otimes \rho_{\mathrm{d}}\right]$. In this way, the spin foam amplitudes of $S O(4) \mathrm{BF}$ theory reduce to 


$$
\begin{aligned}
Z_{B F}(\Delta) & =\sum_{\mathcal{C}_{f}:\{f\} \rightarrow \rho_{f}} \\
& \prod_{f \in \Delta^{\star}} \mathrm{d}_{\rho_{f}} \prod_{e \in \Delta^{\star}} P_{i n v}^{e}\left(\rho_{1}, \cdots, \rho_{\mathrm{d}}\right) .
\end{aligned}
$$

In other words, the $B F$ amplitude associated to a two-complex $\Delta^{\star}$ is simply given by the sum over of all possible assignments of irreducible representations of $G$ to faces of the number obtained by the natural contraction of the network of projectors $P_{i n v}^{e}$, according to the pattern provided defined by the two-complex $\Delta^{\star}$.

There is a nice graphical representation of the partition function of BF theory that will be very useful for some calculations. On the one hand, using this graphical notation one can easily prove the discretization independence of the $\mathrm{BF}$ amplitudes. On the other hand, this graphical notation will simplify the presentation of the new spin foam models of quantum gravity that will be considered in the following sections. This useful notation was introduced by Oeckl $[10,11]$ and used in Ref. [12] to give a general proof of the discretization independence of the BF partition function and the Turaev-Viro invariants for their definition on general cellular decompositions.

We will present this notation in detail: The idea is to represent each representation matrix appearing in (11) by a line (called a wire) labeled by an irreducible representation, and integrations on the group by a box (called a cable). The traces in Eq. (11) imply that there is a wire, labeled by the representation $\rho_{f}$, winding around each face $f \in \Delta^{\star}$. In addition, there is a cable (integration on the group) associated with each edge $e \in \Delta^{\star}$. As in (13), there is a projector $P_{i n v}^{e}$, which is the projector in $\operatorname{Inv}\left[\rho_{1} \otimes \rho_{2} \otimes \cdots \otimes \rho_{\mathrm{d}}\right]$ associated to each edge. This will be represented by a cable with $d$ wires, as shown in (14). Such graphical representation allows for a simple diagrammatic expression of the BF quantum amplitudes.

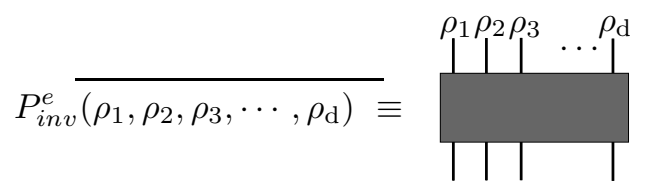

The case of physical interest is $d=4$. In such case, edges are shared by four faces; each cable has now four wires. The cable wire diagram giving the $\mathrm{BF}$ amplitude is dictated by the combinatorics of the dual two complex $\Delta^{\star}$. From Fig. 3, one gets

$$
Z_{B F}(\Delta)=\sum_{\mathcal{C}_{f}:\{f\} \rightarrow \rho_{f}}
$$

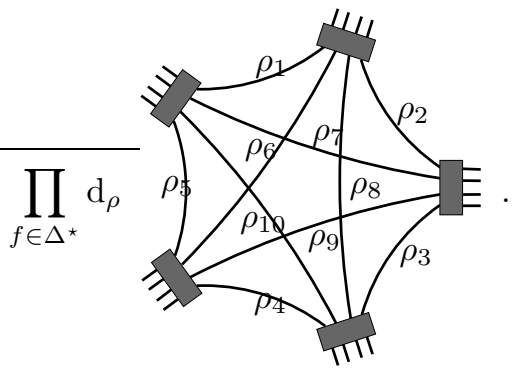

The 10 wires corresponding to the 10 faces $f \in \Delta^{\star}$, sharing a vertex $v \in \Delta^{\star}$, are connected to the neighboring vertices through the 5 cables (representing the projectors in (13) and Fig. 14) associated to the 5 edges $e \in \Delta^{\star}$, sharing the vertex $v \in \Delta^{\star}$.

a. $\quad S U(2) \times S U(2)$ BF theory: a starting point for
4d Riemannian gravity.

We now present the BF quantum amplitudes in the case $G=S U(2) \times S U(2)$. This special case is of fundamental importance in the construction of the gravity models presented in the following sections. The product form of the structure group implies the simple relationship $Z_{B F}(S U(2) \times S U(2))=$ $Z_{B F}(S U(2))^{2}$. Nevertheless, it is important for us to present this example in an explicit way as it will provide the graphical notation that is needed to introduce the gravity models in a simple manner. The spin foam representation of the BF partition function follows from expressing the projectors in (15) in the orthonormal basis of intertwiners, i.e., invariant vectors in $\operatorname{Inv}\left[\rho_{1} \otimes \cdots \otimes \rho_{4}\right]$. From the product form of the structure group, one has

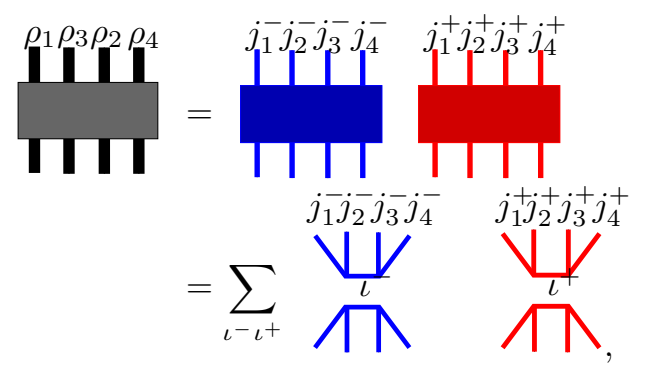


where $\rho_{f}=j_{f}^{-} \otimes j_{f}^{+}, j_{f}^{ \pm}$and $\iota^{ \pm}$are half integers labeling left and right representations of $S U(2)$ that defined the irreducible unitary representations of $G=S U(2) \times S U(2)$. We have used the expression of the right and left $S U(2)$ projectors in a basis of intertwiners, namely

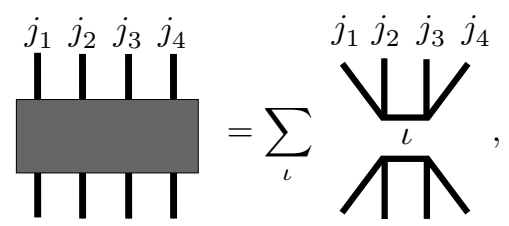

where the four-leg objects on the right hand side denote the invariant vectors spanning a basis of $\operatorname{Inv}\left[j_{1} \otimes \cdots \otimes j_{4}\right]$, and $\iota$ is a half integer, labeling those elements. Accordingly, when replacing the previous expression in (15), one gets

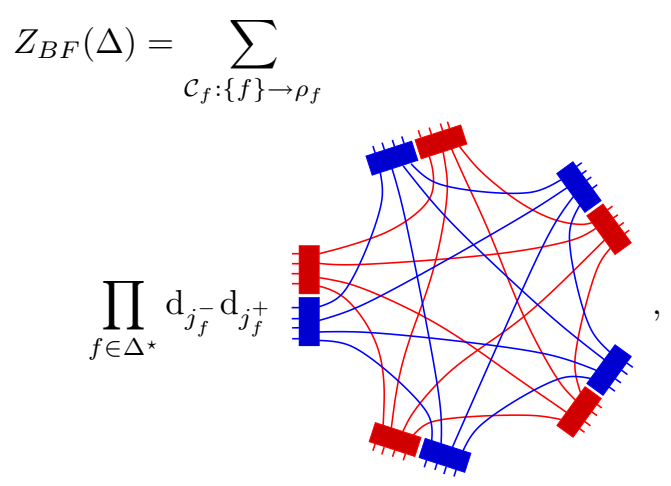

and equivalently,

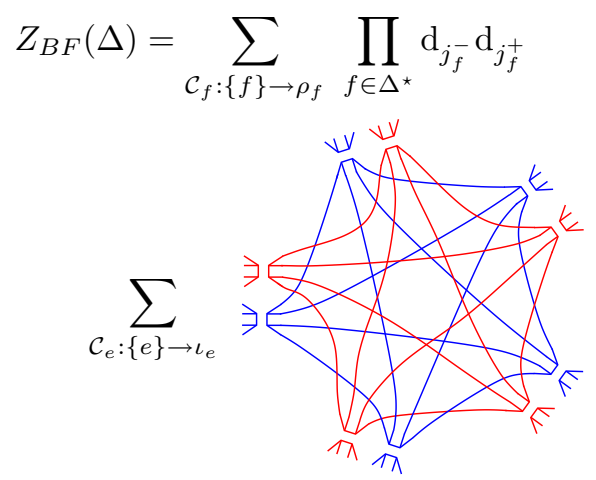

from which we finally obtain the spin foam representation of the $S U(2) \times S U(2)$ partition function as a product of two $S U(2)$ amplitudes, namely

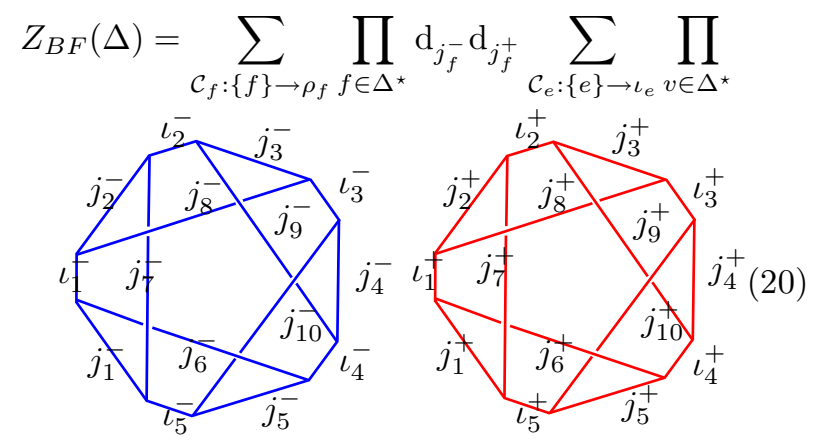

Extra remarks on four-dimensional BF theory

The state sum (11) is generically divergent (due to the gauge freedom analogous to (3)). A regularized version defined in terms of $S U_{q}(2) \times S U_{q}(2)$ was introduced by Crane and Yetter $[13,14]$. As in three dimensions, if an appropriate regularization of bubble divergences is provided, (11) is topologically invariant and the spin foam path integral is discretization independent.

As in the three-dimensional case, BF theory can be coupled to topological defects [15] in any dimension. In the four-dimensional case, defects are string-like [16] and can carry extra degrees of freedom, such as topological Yang-Mills fields [17]. The possibility that quantum gravity could be defined directly from these simple kinds of topological theories has also been considered outside spin foams [18] (for which the UV problem described in the introduction is absent). This is attractive and should, in my view, be considered further.

It is also possible to introduce one-dimensional particles in four-dimensional BF theory and gravity, as shown in Ref. [19].

Two-dimensional BF theory has been used as the basic theory in an attempt to define a manifold independent model of QFT in Ref. [20]. It is also related to gravity in two dimensions in two ways: On the one hand, it is equivalent to the so-called Jackiw-Teitelboim model $[21,22]$, on the other hand it is related to usual $2 \mathrm{~d}$ gravity via constraints in a way similar to the one exploited in four dimensions (see next section). The first relationship has been used in the canonical quantization of the JackiwTeitelboim model in Ref. [23]. The second relationship has been explored in Ref. [24]. 
Papers in Physics, vol. 4, Art. 040004 (2012) / A. Perez

Three-dimensional BF theory and the spin foam quantization presented above are intimately related to classical and quantum gravity in three dimensions (for a classic reference see Ref. [25]). The state sum, as presented above, matches the quantum amplitudes first proposed by Ponzano and Regge in the 60's, based on their discovery of the asymptotic expressions of the $6 \mathrm{j}$ symbols [26], often referred to as the Ponzano-Regge model. Divergences in the above formal expression require regularization. Natural regularizations are available so that the model is well-defined [27-29]. For a detailed study of the divergence structure of the model, see Refs. [30-32]. The quantum deformed version of the above amplitudes lead to the so-called Turaev-Viro model [33], which is expected to correspond to the quantization of threedimensional Riemannian gravity in the presence of a non-vanishing positive cosmological constant. For the definition of observables in the latter context, as well as in the analogue four-dimensional analog, see Ref. [34].

The topological character of BF theory can be preserved by the coupling of the theory with topological defects that play the role of point particles. In the spin foam literature, this has been considered from the canonical perspective in Refs. [35,36] and from the covariant perspective extensively by Freidel and Louapre [37]. These theories have been proved by Freidel and Livine to be dual, in a suitable sense, to certain non-commutative fields theories in three dimensions [38, 39].

Concerning coupling BF theory with nontopological matter, see Refs. $[40,41]$ for the case of fermionic matter, and Ref. [42] for gauge fields. A more radical perspective for the definition of matter in 3d gravity is taken in Ref. [43]. For threedimensional supersymmetric BF theory models, see Refs. [44, 45]

Recursion relations for the $6 \mathrm{j}$ vertex amplitudes have been investigated in Refs. [46,47]. They provide a tool for studying dynamics in spin foams of $3 \mathrm{~d}$ gravity and might be useful in higher dimensions [48].

\section{i. The coherent states representation}

In this section, we introduce the coherent state representation of the $S U(2)$ and $\operatorname{Spin}(4)$ path integral of BF theory. This will be particularly important for the definition of the models defined by Freidel and Krasnov in Ref. [6] that we will address in Section IV as well as in the semiclassical analysis of the new models reported in Section VII. The relevance of such representation for spin foams was first emphasized by Livine and Speziale in Ref. [49].

\section{a. Coherent states}

Coherent states associated with the representation theory of a compact group have been studied by Thiemann and collaborators [50,51,51-59], see also Ref. [60]. Their importance for the new spin foam models was put forward by Livine and Speziale in Ref. [49], where the emphasis was put on coherent states of intertwiners or the so-called quantum tetrahedron (see also [61]). Here we follow the presentation of [6].

In order to build coherent states for $\operatorname{Spin}(4)$, we start by introducing them in the case of $S U(2)$. Starting from the representation space $\mathscr{H}_{j}$ of dimension $\mathrm{d}_{j} \equiv 2 j+1$, one can write the resolution of the identity in terms of the canonical orthonormal basis $|j, m\rangle$ as

$$
1_{j}=\sum_{m}|j, m\rangle\langle j, m|
$$

where $-j \leq m \leq j$. There exists an over complete basis $|j, g\rangle \in \mathscr{H}_{j}$, labeled by $g \in S U(2)$, such that

$$
1_{j}=\mathrm{d}_{j} \int_{\mathrm{SU}(2)} d g|j, g\rangle\langle j, g|,
$$

The states $|j, g\rangle \in \mathscr{H}_{j}$ are $S U(2)$ coherent states defined by the action of the group on maximum weight states $|j, j\rangle$ (themselves coherent), namely

$$
|j, g\rangle \equiv g|j, j\rangle=\sum_{m}|j, m\rangle D_{m j}^{j}(g),
$$

where $D_{m j}^{j}(g)$ are the matrix elements of the unitary representations in the $|j, m\rangle$ (Wigner matrices). Equation (22) follows from the orthonormality of unitary representation matrix elements, namely 
PApers in Physics, vol. 4, ART. 040004 (2012) / A. Perez

$$
\begin{aligned}
& \mathrm{d}_{j} \int_{\mathrm{SU}(2)} d g|j, g\rangle\langle j, g|, \\
& =\mathrm{d}_{j} \sum_{m m^{\prime}}|j, m\rangle\left\langle j, m^{\prime}\right| \int_{\mathrm{SU}(2)} d g D_{m j}^{j}(g) \overline{D_{m^{\prime} j}^{j}(g)} \\
& =\sum_{m}|j, m\rangle\langle j, m|,
\end{aligned}
$$

where in the last equality we have used the orthonormality of the matrix elements. The decomposition of the identity (22) can be expressed as an integral on the two-sphere of directions $S^{2}=$ $S U(2) / U(1)$ by noticing that $D_{m j}^{j}(g)$ and $D_{m j}^{j}(g h)$ differ only by a phase for any group element $h$ from a suitable $U(1) \subset S U(2)$. Thus, one has

$$
1_{j}=\mathrm{d}_{j} \int_{S^{2}} d n|j, n\rangle\langle j, n|,
$$

where $n \in S^{2}$ is integrated with the invariant measure of the sphere. The states $|j, n\rangle$ form (an overcomplete) basis in $\mathscr{H}_{j}$. $S U(2)$ coherent states have the usual semiclassical properties. Indeed, if one considers the generators $J^{i}$ of $s u(2)$, one has

$$
\left\langle j, n\left|\hat{J}^{i}\right| j, n\right\rangle=j n^{i},
$$

where $n^{i}$ is the corresponding three-dimensional unit vector for $n \in S^{2}$. The fluctuations of $\hat{J}^{2}$ are also minimal with $\Delta J^{2}=\hbar^{2} j$, where we have restored $\hbar$ for clarity. The fluctuations go to zero in the limit $\hbar \rightarrow 0$ and $j \rightarrow \infty$, while $\hbar j$ is kept constant. This kind of limit will be used often as a notion of semiclassical limit in spin foams. The state $|j, n\rangle$ is a semiclassical state describing a vector in $\mathbb{R}^{3}$ of length $j$ and of direction $n$. It will be convenient to introduce the following graphical notation for Eq. (25)

$$
j \mid=\mathrm{d}_{j} \int_{S^{2}} d n \quad \begin{aligned}
& j \\
& \vdots \\
& n
\end{aligned}
$$

Finally, an important property of $S U(2)$ coherent states stemming from the fact that

$$
|j, j\rangle=\left|\frac{1}{2}, \frac{1}{2}\right\rangle\left|\frac{1}{2}, \frac{1}{2}\right\rangle \cdots\left|\frac{1}{2}, \frac{1}{2}\right\rangle \equiv\left|\frac{1}{2}, \frac{1}{2}\right\rangle^{\otimes 2 j}
$$

is that

$$
|j, n\rangle=\left|\frac{1}{2}, n\right\rangle^{\otimes 2 j} .
$$

The above property will be of key importance in constructing effective discrete actions for spin foam models. In particular, it will play a central role in the study of the semiclassical limit of the EPRL and FK models studied in Sections III, and IV. In the following subsection, we provide an example for $\operatorname{Spin}(4)$ BF theory.

b. Spin(4) BF theory: Amplitudes in the coherent state basis

Here we study the coherent states representation of the path integral for Spin(4) BF theory. The construction presented here can be extended to more general cases. The present case is, however, of particular importance for the study of gravity models presented in Sections III, and IV. With the introduction of coherent states, one achieves the most difficult part of the work. In order to express the Spin(4) BF amplitude in the coherent state representation, one simply inserts a resolution of the identity in the form (25) on each and every wire connecting neighboring vertices in the expression (18) for the BF amplitudes. The result is

$$
\begin{gathered}
Z_{B F}(\Delta)=\sum_{\mathcal{C}_{f}:\{f\} \rightarrow \rho_{f}} \prod_{f \in \Delta^{\star}} \mathrm{d}_{j_{f}^{-}} \mathrm{d}_{j_{f}^{+}} \\
\int \prod_{e \in \in \Delta^{*}} \mathrm{~d}_{j_{e f}^{-}} \mathrm{d}_{j_{e f}^{+}} d n_{e f}^{-} d n_{e f}^{+}
\end{gathered}
$$

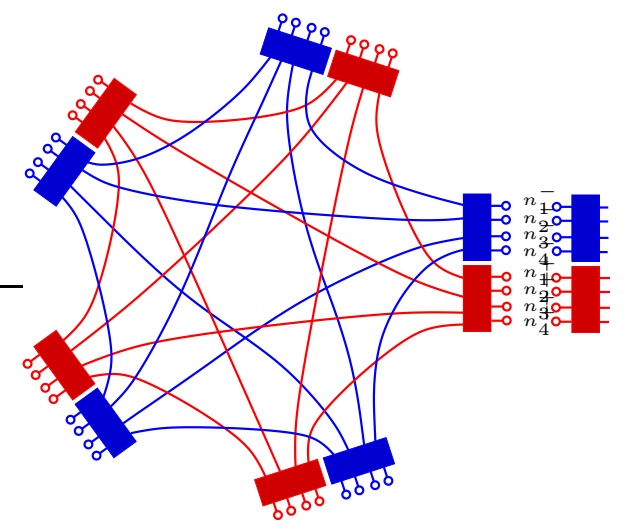

where we have explicitly written the $n_{ \pm} \in S^{2}$ integration variables only on a single cable. One observes that there is one $n_{ \pm} \in S^{2}$ per each wire coming out at an edge $e \in \Delta^{\star}$. As wires are in one-to-one correspondence with faces $f \in \Delta^{\star}$, the integration variables $n_{\text {ef }}^{ \pm} \in S^{2}$ are labeled by an 
Papers in Physics, vol. 4, Art. 040004 (2012) / A. Perez

edge and face subindex. In order to get an expression of the BF path integral in terms of an affective action, we restore, at this stage, the explicit group integrations represented by the boxes in the previous equation. One gets

$$
\begin{gathered}
Z_{B F}(\Delta)=\sum_{\mathcal{C}_{f}:\{f\} \rightarrow \rho_{f}} \prod_{f \in \Delta^{\star}} \mathrm{d}_{j_{f}^{-}} \mathrm{d}_{j_{f}^{+}} \int \\
\prod_{e \in \Delta^{\star}} \mathrm{d}_{j_{e f}^{-}} \mathrm{d}_{j_{e f}^{+}} d n_{e f}^{-} d n_{e f}^{+} \prod_{v \in \Delta^{\star}} \prod_{e, e^{\prime} \in v} \\
d g_{e f}^{-} d g_{e f}^{+}\left(\left\langle n_{e f}^{-}\left|\left(g^{-}\right)_{e f}^{-1} g_{e^{\prime} f}^{-}\right| n_{e^{\prime} f}^{-}\right\rangle\right)^{2 j_{f}^{-}} \\
\left(\left\langle n_{e f}^{+}\left|\left(g^{+}\right)_{e f}^{-1} g_{e^{\prime} f}^{+}\right| n_{e^{\prime} f}^{+}\right\rangle\right)^{2 j_{f}^{+}}
\end{gathered}
$$

where we have used the coherent states property (28), and $\left|n^{ \pm}\right\rangle$is a simplified notation for $\left|\frac{1}{2}, n^{ \pm}\right\rangle$. The previous equation can be finally written as

$$
\begin{gathered}
Z_{B F}(\Delta)=\sum_{\mathcal{C}_{f}:\{f\} \rightarrow \rho_{f}} \prod_{f \in \Delta^{\star}} \mathrm{d}_{j_{f}^{-}} \mathrm{d}_{j_{f}^{+}} \int \\
\prod_{e \in \Delta^{\star}} \mathrm{d}_{j_{e f}^{-}} \mathrm{d}_{j_{e f}^{+}} d n_{e f}^{-} d n_{e f}^{+} d g_{e f}^{-} d g_{e f}^{+} \\
\exp \left(S_{j^{ \pm}, \mathbf{n}^{ \pm}}^{d}\left[g^{ \pm}\right]\right),
\end{gathered}
$$

where the discrete action

$$
S_{j^{ \pm}, \mathbf{n}^{ \pm}}^{d}\left[g^{ \pm}\right]=\sum_{v \in \Delta^{\star}} S_{j_{v}, \mathbf{n}_{v}}^{v}\left[g^{ \pm}\right]
$$

with

$$
S_{j, \mathbf{n}}^{v}[g]=\sum_{a<b=1}^{5} 2 j_{a b} \ln \left\langle n_{a b}\left|g_{a}^{-1} g_{b}\right| n_{b a}\right\rangle,
$$

and the indices $a, b$ label the five edges of a given vertex. The previous expression is equal to the form (11) of the BF amplitude. In the case of the gravity models studied in what follows, the coherent state path integral representation will be the basic tool for the study of the semiclassical limit of the models and the relationship with Regge discrete formulation of general relativity.

\section{ii. The relationship between gravity and BF theory}

The field theory described in the present section has no local degrees of freedom. It represents the simplest example of a topological field theory in four dimensions. The interest of this theory for gravity models stems from the fact that an action for the gravitational degrees of freedom (basically equivalent to general relativity in the first order formulation) can be obtained by supplementing a $4 \mathrm{~d} B F$ theory action with internal gauge group $S L(2, \mathbb{C})$ (Lorentzian) or Spin(4) (Riemannian) with the following set of quadratic constraints on the $B$-field

$$
\epsilon_{I J K L} B_{\mu \nu}^{I J} B_{\rho \sigma}^{K L}-e \epsilon_{\mu \nu \rho \sigma} \approx 0,
$$

where $e \equiv \sigma^{2}(1 / 4 !) \epsilon_{I J K L} B_{\mu \nu}^{I J} B_{\rho \sigma}^{K L} \epsilon^{\mu \nu \rho \sigma}$ where $\sigma^{2}= \pm 1$ according to whether one is in the Riemannian or Lorentzian case. More generally, a oneparameter family of gravity actions can be obtained from the imposition of the previous constraints on the following modified $\mathrm{BF}$ action

$$
S_{\gamma}(B, \omega)=\int_{\mathcal{M}}\left\langle\left({ }^{\star} B+\frac{1}{\gamma} B\right) \wedge F(\omega)\right\rangle,
$$

where $\gamma$ is the Immirzi parameter. The strategy behind the definition of the new spin foam models for quantum gravity consists of imposing these constraints on the path integral of BF theory on the momenta $J={ }^{\star} B+\frac{1}{\gamma} B$ conjugated to $\omega$. In order to impose the Plebanski constraints above, it will be convenient to express the $B$ field in terms of the momenta $J$, namely

$$
B=\frac{\gamma}{1-\sigma^{2} \gamma^{2}}\left(J-\gamma^{\star} J\right) .
$$

The imposition of the constraints (34) on the BF path integral on a fixed discretization can be done in two different ways: by directly restricting the spin foam configurations (this is the EPRL approach described in the following section), or by restricting the semiclassical values of the $B$ field in the coherent state representation of the BF path integral (this is the FK strategy described in Section IV).

\section{The Engle-Pereira-Rovelli- Livine (EPRL) model}

In this section, we introduce the Engle-PereiraRovelli-Livine (EPRL) model $[4,5]$. The section is organized as follows: The relevant representation theory is introduced in Subsection i. In Subsection ii, we present and discuss the linear simplicity constraints - classically equivalent to the Plebanski constraints - and discuss their implementation in 
Papers in Physics, vol. 4, Art. 040004 (2012) / A. Perez

the quantum theory. In Subsection iii, we introduce the EPRL model of Riemannian gravity. In Subsection iv, we prove the validity of the quadratic Plebanski constraints - reducing BF theory to general relativity - directly in the spin foam representation. In Subsection v, we present the coherent state representation of the Riemannian EPRL model. In Subsection vi, we describe the Lorentzian model. The material of this section will also allow us to describe the construction of the closely related (although derived from a different logic) Riemannian FK constructed in Ref. [6]. The idea that linear simplicity constraints are more convenient for dealing with the constraints that reduce BF theory to gravity was pointed out by Freidel and Krasnov in this last reference.

\section{i. Representation theory of $\operatorname{Spin}(4)$ and $S L(2, \mathbb{C})$ and the canonical basis}

In this section, we present the representation theory of the groups $\operatorname{Spin}(4)$ and $S L(2, \mathbb{C})$ that is necessary for the definition of the new spin foam models for Riemannian and Lorentzian gravity, respectively. To emphasize the highly symmetric structure of the two, we present them in a unified notation where a parameter $\sigma=1$ for the Riemannian sector and $\sigma=i$ for the Lorentzian one. The simple relationship between the two might be a hint to a possible relationship between model amplitudes in a spirit similar to the interesting link between Euclidean and Lorentzian QFT provided by Wick rotations ${ }^{1}$. Unitary irreducible representations $\mathscr{H}_{p, k}$ of $\operatorname{Spin}(4)$ and $S L(2, \mathbb{C})$ are labeled by two parameters, $p$ and $k$. In the case of $\operatorname{Spin}(4)=S U(2) \times S U(2)$, the unitary irreducible representations are finite-dimensional and the labels $p$ and $k$ can be expressed in terms of the half integers labeling the right and left $S U(2)$ unitary representations $j^{ \pm}$, as follows

$$
p=j^{+}+j^{-}+1 \quad k=\left|j^{+}-j^{-}\right| .
$$

In the $S L(2, \mathbb{C})$ case, the unitary irreducible representations are infinite-dimensional and one has

$$
p \in \mathbb{R}^{+} \quad k \in \mathbb{N} / 2 .
$$

The two Casimirs are $C_{1}=\frac{1}{2} J_{I J} J^{I J}=L^{2}+\sigma^{2} K^{2}$ and $C_{2}=\frac{1}{2}{ }^{\star} J_{I J} J^{I J}=K \cdot L$ where $L^{i}$ are the generators of an arbitrary rotation subgroup and $K^{i}$ are the generators of the corresponding boosts. The Casimirs act on $|p, k\rangle \in \mathscr{H}_{p, k}$, as follows

$$
\begin{aligned}
& C_{1}|p, k\rangle=\frac{1}{2}\left(k^{2}+\sigma^{2} p^{2}-1\right)|p, k\rangle \\
& C_{2}|p, k\rangle=p k|p, k\rangle .
\end{aligned}
$$

For details on the representation theory of $S L(2, \mathbb{C})$, see Refs. [63-65]. The definition of the EPRL model requires the introduction of an (arbitrary) subgroup $S U(2) \subset \operatorname{Spin}(4)$ or $S U(2) \subset$ $S L(2, \mathbb{C})$, according to whether one is working in the Riemannian or in the Lorentzian sector. This subgroup corresponds to the internal gauge group of the gravitational phase space in connection variables in the time gauge (see Ref. [8] for details). Hence, in the quantum theory, the representation theory of this $S U(2)$ subgroup will be important. This importance will soon emerge as apparent from the imposition of the constraints that define the EPRL model. The link between the unitary representations of $S L(2, \mathbb{C})$ and those of $S U(2)$ is given by the decomposition

$$
\mathscr{H}_{p, k}=\bigoplus_{j=k}^{p-1} \mathscr{H}_{j}=\bigoplus_{j=\left|j^{+}-j^{-}\right|}^{j^{+}+j^{-}} \mathscr{H}_{j},
$$

for the Riemannian sector, and

$$
\mathscr{H}_{p, k}=\bigoplus_{j=k}^{\infty} \mathscr{H}_{j},
$$

for the Lorentzian sector. As the unitary irreducible representations of the subgroup $S U(2) \in$ $\operatorname{Spin}(4)$ and $S U(2) \in S L(2, \mathbb{C})$ are essential for understanding the link of the EPRL model and the operator canonical formulation of LQG, it will be convenient to express the action of the generators of the Lie algebra of the corresponding group in a basis adapted to the above equation. In order to do this, we first notice that the Lie algebra $\operatorname{spin}(4)$ and $s l(2, \mathbb{C})$ can be characterized in terms of the generators of a rotation subgroup $L^{i}$ and the remaining

\footnotetext{
${ }^{1}$ Such explicit relationship between gravity amplitudes in the Euclidean and Lorentzian sectors can be established by analytic continuation in $3 \mathrm{~d}[62]$.
} 
Papers in Physics, vol. 4, ART. 040004 (2012) / A. Perez

boost generators $K^{i}$, as follows

$$
\begin{aligned}
& {\left[L_{3}, L_{ \pm}\right]= \pm L_{ \pm}\left[L_{+}, L_{-}\right]=2 L_{3}} \\
& {\left[L_{+}, K_{+}\right]=\left[L_{-}, K_{-}\right]=\left[L_{3}, K_{3}\right]=0} \\
& {\left[K_{3}, L_{ \pm}\right]= \pm K_{ \pm}\left[L_{ \pm}, K_{\mp}\right]= \pm 2 K_{3}} \\
& {\left[L_{3}, K_{ \pm}\right]= \pm K_{ \pm}} \\
& {\left[K_{3}, K_{ \pm}\right]= \pm \sigma^{2} L_{ \pm}} \\
& {\left[K_{+}, K_{-}\right]=2 \sigma^{2} L_{3},}
\end{aligned}
$$

where $K_{ \pm}=K^{1} \pm i K^{2}$ and $L_{ \pm}=L^{1} \pm i L^{2}$, respectively. The action of the previous generators in the basis $|p, k ; j, m\rangle$ can be shown to be

$$
\begin{aligned}
L^{3}|p, k ; j, m\rangle & =m|p, k ; j, m\rangle, \\
L^{+}|p, k ; j, m\rangle & =\sqrt{(j+m+1)(j-m)} \\
& |p, k ; j, m+1\rangle, \\
L^{-}|p, k ; j, m\rangle & =\sqrt{(j+m)(j-m+1)} \\
& |p, k ; j, m-1\rangle, \\
K^{3}|p, k ; j, m\rangle & =\alpha_{j} \sqrt{j^{2}-m^{2}}|p, k ; j-1, m\rangle \\
& +\gamma_{j} m|p, k ; j, m\rangle \\
& -\alpha_{j+1} \sqrt{(j+1)^{2}-m^{2}} \\
& |p, k ; j+1, m\rangle, \\
K^{+}|p, k ; j, m\rangle & =\alpha_{j} \sqrt{(j-m)(j-m-1)} \\
& |p, k ; j-1, m+1\rangle \\
& +\gamma_{j} \sqrt{(j-m)(j+m+1)} \\
& |p, k ; j, m+1\rangle \\
& +\alpha_{j+1} \sqrt{(j+m+1)(j+m+2)} \\
& |p, k ; j+1, m+1\rangle, \\
& =-\alpha_{j} \sqrt{(j+m)(j+m-1)} \\
& |p, k ; j-1, m-1\rangle \\
& +\gamma_{j} \sqrt{(j+m)(j-m+1)} \\
& |p, k ; j, m-1\rangle \\
& -\alpha_{j+1} \sqrt{(j-m+1)(j-m+2)} \\
& |p, k ; j+1, m-1\rangle,
\end{aligned}
$$

where

$$
\gamma_{j}=\frac{k p}{j(j+1)}, \quad \alpha_{j}=\sigma \sqrt{\frac{\left(j^{2}-k^{2}\right)\left(j^{2}+p^{2}\right)}{j^{2}\left(4 j^{2}-1\right)}}
$$

The previous equations will be important in what follows: they will allow for the characterization of the solutions of the quantum simplicity constraints, in both the Riemannian and Lorentzian models, in a direct manner. This concludes the review of the representation theory that is necessary for the definition of the EPRL model.

\section{ii. The linear simplicity constraints}

As first shown in Ref. [6], the quadratic Plebanski simplicity constraints - and more precisely in their dual version presented below (34) - are equivalent in the discrete setting to the linear constraint on each face of a given tetrahedron

$$
D_{f}^{i}=L_{f}^{i}-\frac{1}{\gamma} K_{f}^{i} \approx 0,
$$

where the label $f$ makes reference to a face $f \in$ $\Delta^{\star}$, and where (very importantly) the subgroup $S U(2) \subset \operatorname{Spin}(4)$ (or $S L(2, \mathbb{C})$ ) that is necessary for the definition of the above constraints is chosen arbitrarily at each tetrahedron, equivalent on each edge $e \in \Delta^{\star}$. Such choice of the rotation subgroup is the precise analog of the time gauge in the canonical analysis of general relativity. The EPRL model is defined by imposing the previous constraints as operator equations on the Hilbert spaces defined by the unitary irreducible representations of the internal gauge group that take part in the state-sum of BF theory. We will show in Subsection iv that the models constructed on the requirement of a suitable imposition of the linear constraints (45) satisfy the usual quadratic Plebanski constraints - that reduce BF theory to general relativity - in the path integral formulation (up to quantum corrections which are neglected in the usual semiclassical limit).

From the commutation relations (42), from previous section, we can easily compute the commutator of the previous tetrahedron constraints and conclude that, in fact, it does not close, namely

$$
\begin{aligned}
{\left[D_{f}^{i}, D_{f^{\prime}}^{j}\right] } & =\delta_{f f^{\prime}} \epsilon_{k}^{i j}\left[\left(1+\frac{\sigma^{2}}{\gamma^{2}}\right) L_{f}^{k}-\frac{2}{\gamma} K_{e}^{k}\right] \\
& =2 \delta_{e e^{\prime}} \epsilon_{k}^{i j} D^{k}+\delta_{e e^{\prime}} \frac{\sigma^{2}-\gamma^{2}}{\gamma^{2}} \epsilon_{k}^{i j} L_{f}^{k} .
\end{aligned}
$$

The previous commutation relations imply that the constraint algebra is not closed and cannot therefore be imposed as operator equations on the states summed over in the BF partition function in general. There are two interesting exceptions to the previous statement: 
Papers in Physics, vol. 4, ART. 040004 (2012) / A. Perez

1. The first one is to take $\gamma= \pm \sigma$. This corresponds to the description of the model in terms of self-dual or anti-self-dual variables. Unfortunately, the construction of the new models is not well defined in this case for the Lorentzian theory and leads to a trivial result in the Riemannian sector: $S U(2)$ BF theory.

2. The second possibility is to work in the sector where $L_{f}^{i}=0$. This choice leads to the BarretCrane model [66], where the degrees of freedom of BF theory seem over constrained: Boundary states satisfying the $\mathrm{BC}$ constraints are a very small subset of the allowed boundary states in LQG. This is believed to be problematic if gravity is to be recovered at low energies.

The EPRL model is obtained by restricting the representations appearing in the expression of the $\mathrm{BF}$ partition function so that at each tetrahedron the linear constraint (45) is the strongest possible way that is compatible with the uncertainties relations stemming from (46). In addition, one would add the requirement that the state-space of the tetrahedra is compatible with the state-space of the analogous excitation in the canonical context of LQG, so that arbitrary states in the kinematical state of LQG have non-trivial amplitudes in the model.

Due to the fact that the constraints $D_{f}^{i}$ do not form a closed (first class) algebra in the generic case, one needs to devise a weaker sense in which they are to be imposed. One possibility is to consider the Gupta-Bleuler criterion consisting of selecting a suitable class of states for which the matrix elements on $D_{f}^{i}$ vanish. One notices from (43) that if we chose the subspace $\mathscr{H}_{j} \subset \mathscr{H}_{p, k}$, we would have

$$
\begin{array}{r}
\left\langle p, k, j, q\left|D_{f}^{3}\right| p, k, j, m\right\rangle=\delta_{q, m} m\left(1-\frac{\gamma_{j}}{\gamma}\right) \\
\left\langle p, k, j, q\left|D_{f}^{ \pm}\right| p, k, j, m\right\rangle=\delta_{q \pm 1, m} \\
\times \sqrt{(j \pm m+1)(j \mp m)}\left(1-\frac{\gamma_{j}}{\gamma}\right) .
\end{array}
$$

The matrix elements of the linear constraints vanish in this subclass if one chooses

$$
\gamma_{j}=\frac{p k}{j(j+1)}=\gamma
$$

There are two cases:
1. Case $\gamma<1$ : Following Ref. [67], in this case one restricts the representations to

$$
\begin{aligned}
& \text { Riemannian: } p=j+1, k=\gamma j . \\
& \text { Lorentzian: } p=\gamma(j+1), k=j .
\end{aligned}
$$

which amounts to choosing the maximum weight component $j=p-1$ in the expansion (41). In the Riemannian case, the above choice translates into $j^{ \pm}=(1 \pm \gamma) j / 2$ for the $\mathrm{SU}(2)$ right and left representations. Notice that the solutions to the simplicity constraints in the Riemannian and Lorentzian sectors look very different for $\gamma<1$. Simple algebra shows that condition (47) is met. There are indeed other solutions [68] to the Gupta-Bleuler criterion in this case.

2. Case $\gamma>1$ : In this case, according to Ref. [69], one restricts the representations to

$$
\begin{gathered}
\text { Riemannian: } p=\gamma(j+1), k=j . \\
\text { Lorentzian: } p=\gamma(j+1), k=j .
\end{gathered}
$$

which amounts to choosing the minimum weight component $j=k$ in the expansion (41). For the Riemannian case, we can write the solutions in terms of $j^{ \pm}=(\gamma \pm 1) \frac{j}{2}+\frac{\gamma-1}{2}$. Notice that for $\gamma>1$ there is complete symmetry between the solutions of the Riemannian and Lorentzian sectors. In my opinion, this symmetry deserves further investigation as it might be an indication of a deeper connection between the Riemannian and Lorentzian models (again, such relationship is a fact in $3 \mathrm{~d}$ gravity [62].

Another criterion for weak imposition can be developed by studying the spectrum of the Master constraint $M_{f}=D_{f} \cdot D_{f}$. Strong imposition of the constraints $D_{f}^{i}$ would amount to looking for the kernel of the master constraint $M_{f}$. However, generically, the positive operator associated with the master constraint does not contain the zero eigenvalue in the spectrum due to the open nature of the constraint algebra (46).

It is convenient, as in Ref. [70], to express the master constraint in a manifestly invariant way. In order to get a gauge invariant constraint one starts 
from the master constraint and uses the $D_{f}^{i}=0$ classically to write it in terms of Casimirs, namely

$$
M_{f}=\left(1+\sigma^{2} \gamma^{2}\right) C_{2}-2 C_{1} \gamma,
$$

where $C_{1}$ and $C_{2}$ are the Casimirs given in Eq. (39). The minimum eigenvalue condition is

$$
\begin{gathered}
\text { Riemannian: } p=j, k=\gamma j . \\
\text { Lorentzian: } p=\gamma j, k=j .
\end{gathered}
$$

The minimum eigenvalue is $m_{\min }=\hbar^{2} \gamma j\left(\gamma^{2}-1\right)$ for the Riemannian case and $m_{\min }=\gamma$ for the Lorentzian case. The master constraint criterion works better in the Lorentzian case, as pointed out in Ref. [70]. More recently, it has been shown that the constraint solutions $p=\gamma j$ and $k=j$ also follow naturally from a spinor formulation of the simplicity constraints [71-73]. The above criterion is used in the definition of the EPRL model.

It is important to point out that the Riemannian case imposes strong restrictions on the allowed values of the Immirzi parameter if one wants the spin $j \in \mathbb{N} / 2$ to be arbitrary (in order to have all possible boundary states allowed in LQG). In this case, the only possibilities are $\gamma=\mathbb{N}$ or $\gamma=1$. This restriction is not natural from the viewpoint of LQG. Its relevance, if any, remains mysterious at this stage.

Summarizing, in the Lorentzian (Riemannian) EPRL model one restricts the $S L(2, \mathbb{C})(\operatorname{Spin}(4))$ representations of $\mathrm{BF}$ theory to those satisfying

$$
p=\gamma j \quad k=j
$$

for $j \in \mathbb{N} / 2$. From now on, we denote the subset of admissible representation

$$
\mathscr{K}_{\gamma} \subset \operatorname{Irrep}(S L(2, \mathbb{C}))(\operatorname{Irrep}(\operatorname{Spin}(4)))
$$

The admissible quantum states $\Psi$ are elements of the subspace $\mathscr{H}_{j} \subset \mathscr{H}_{\gamma j, j}$ (i.e., minimum weight states) which satisfy the constraints (45) in the following semiclassical sense:

$$
\left(K_{f}^{i}-\gamma L_{f}^{i}\right) \Psi=\mathscr{O}_{s c},
$$

where the symbol $\mathscr{O}_{s c}$ (order semiclassical) denotes a quantity that vanishes in limit $\hbar \rightarrow 0, j \rightarrow \infty$ with $\hbar j=$ constant. In the Riemannian case, the previous equation can be written as

$$
\left[(1-\gamma) J_{+}^{i}-(1+\gamma) J_{-}^{i}\right] \Psi=\mathscr{O}_{s c},
$$

which in turn has a simple graphical representation in terms of spin-network grasping operators, namely

$$
-(1+\gamma) \underbrace{k}_{J_{+}}+\left.(1-\gamma)\right|_{J_{-}} ^{k}=\mathscr{O}_{s c}
$$

The previous equation will be of great importance in the graphical calculus that will allow us to show that the linear constraint imposed here, at the level of states, implies the vanishing of the quadratic Plebanski constraints (34) and their fluctuations, computed in the path integral sense, in the appropriate large spin semiclassical limit.

\section{iii. Presentation of the Riemannian EPRL amplitude}

Here we complete the definition of the EPRL models by imposing the linear constraints on the BF amplitudes constructed in Section II. We will also show that the path-integral expectation value of the Plebanski constraints (34), as well as their fluctuations, vanish in a suitable semiclassical sense. This shows that the EPRL model can be considered as a lattice definition of the a quantum gravity theory.

We start with the Riemannian model for which a straightforward graphical notation is available. The first step is the translation of Eq. (40) for $p$ and $k$ satisfying the simplicity constraintsin terms of the graphical notation introduced in Section II. Concretely, for $\gamma<1$, one has $j^{ \pm}=$ $(1 \pm \gamma) j / 2 \in \mathscr{K}_{\gamma}$ and (40) becomes

$$
(1-\gamma) \frac{j}{2}||(1+\gamma) \frac{j}{2} \bigoplus_{\alpha=\gamma j}^{j}
$$

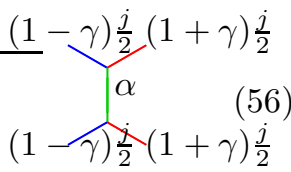

For $\gamma>1$ we have

$$
(\gamma-1) \frac{j}{2}||(1+\gamma) \frac{j}{2} \bigoplus_{\alpha=j}^{\gamma j} \quad \begin{aligned}
& (\gamma-1) \frac{j}{2}(1+\gamma) \frac{j}{2} \\
& \alpha-1) \frac{\dot{\alpha}}{2}(1+\gamma) \frac{j}{2}
\end{aligned}
$$


Papers in Physics, vol. 4, Art. 040004 (2012) / A. Perez

The implementation of the linear constraints of Subsection ii consists of restricting the representations $\rho_{f}$ of $\operatorname{Spin}(4)$ (appearing in the state sum amplitudes of BF theory, as written in Eq. (18)) to the subclass $\rho_{f} \in \mathscr{K}_{\gamma} \subset \operatorname{Irrep}(\operatorname{Spin}(4))$, defined above, while projecting to the highest weight term in (56) for $\gamma<1$. For $\gamma>1$, one must take the minimum weight term in (57). The action of this projection will be denoted $\mathscr{Y}_{j}: \mathscr{H}_{(1+\gamma) j / 2,|(1-\gamma)| j / 2} \rightarrow \mathscr{H}_{j}$, graphically

$$
\mathscr{Y}_{j}\left[|\gamma-1| \frac{j}{2}||(1+\gamma) \frac{j}{2}\right]=\gamma^{j} .
$$

Explicitly, one takes the expression of the BF partition function (13) and modifies it by replacing the projector $P_{i n v}^{e}\left(\rho_{1}, \cdots, \rho_{4}\right)$ with $\rho_{1}, \cdots \rho_{4} \in \mathscr{K}_{\gamma}$ by a new object

$$
\begin{aligned}
& P_{e p r l}^{e}\left(j_{1}, \cdots, j_{4}\right) \equiv P_{i n v}^{e}\left(\rho_{1} \cdots \rho_{4}\right) \\
& \times\left(\mathscr{Y}_{j_{1}} \otimes \cdots \otimes \mathscr{Y}_{j_{4}}\right) P_{i n v}^{e}\left(\rho_{1} \cdots \rho_{4}\right)
\end{aligned}
$$

with $j_{1}, \cdots j_{4} \in \mathbb{N} / 2$, implementing the linear constraints described in the previous section. Graphically, the modification of BF theory that produces the EPRL model corresponds to the replacement

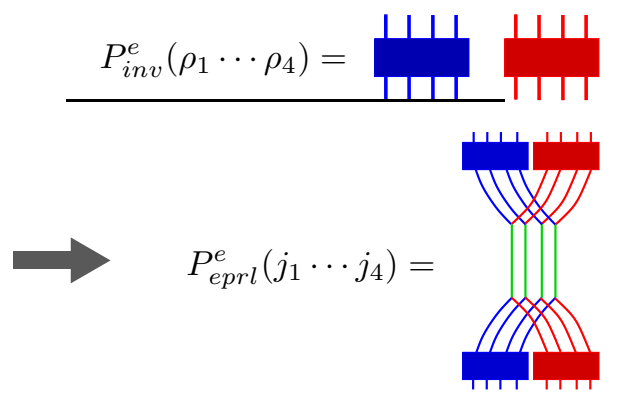

on the expression (18), where we have dropped the representation labels from the figure for simplicity. We have done the operation (58) on each an every of the four pairs of representations. The $\operatorname{Spin}(4)$ integrations represented by the two boxes at the top and bottom of the previous graphical expression restore the full $\operatorname{Spin}(4)$ invariance as the projection (58) breaks this latter symmetry for being based on the selection of a special subgroup $S U(2) \subset \operatorname{Spin}(4)$ in its definition (see Subsection c for an important implication). One should simply keep in mind that green wires in the previous two equations and in the ones that follow are labeled by arbitrary spins $j$ (which are being summed over in the expression of the amplitude (61)), while red and blue wires are labeled by $j^{+}=(1+\gamma) j / 2$ and $j^{-}=|1-\gamma| j / 2$, respectively. With this, (18) is modified to

$$
\begin{aligned}
Z_{e p r l}^{E}(\Delta)= & \sum_{\rho_{f} \in \mathscr{K}} \prod_{f \in \Delta^{\star}} \mathrm{d}_{|1-\gamma| \frac{j}{2}} \mathrm{~d}_{(1+\gamma) \frac{j}{2}} \\
& \times \prod_{e} P_{e p r l}^{e}\left(j_{1}, \cdots, j_{4}\right)= \\
= & \sum_{\rho_{f} \in \mathscr{K}} \prod_{f \in \Delta^{\star}} \mathrm{d}_{|1-\gamma| \frac{j}{2}} \mathrm{~d}_{(1+\gamma) \frac{j}{2}}
\end{aligned}
$$

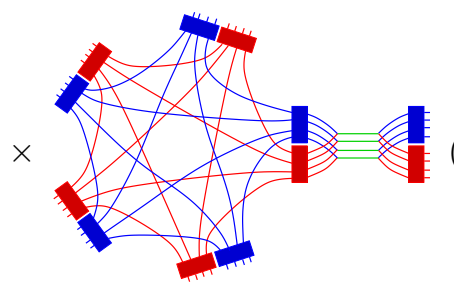

The previous expression defines the EPRL model amplitude.

a. The spin foam representation of the EPRL amplitude

Now we will work out the spin foam representation of the EPRL amplitude which, at this stage, will take no more effort than the derivation of the spin foam representation for $\operatorname{Spin}(4)$ BF theory, as we went from Eq. (18) to Eq. (20) in Section II. The first step is given in the following equation 

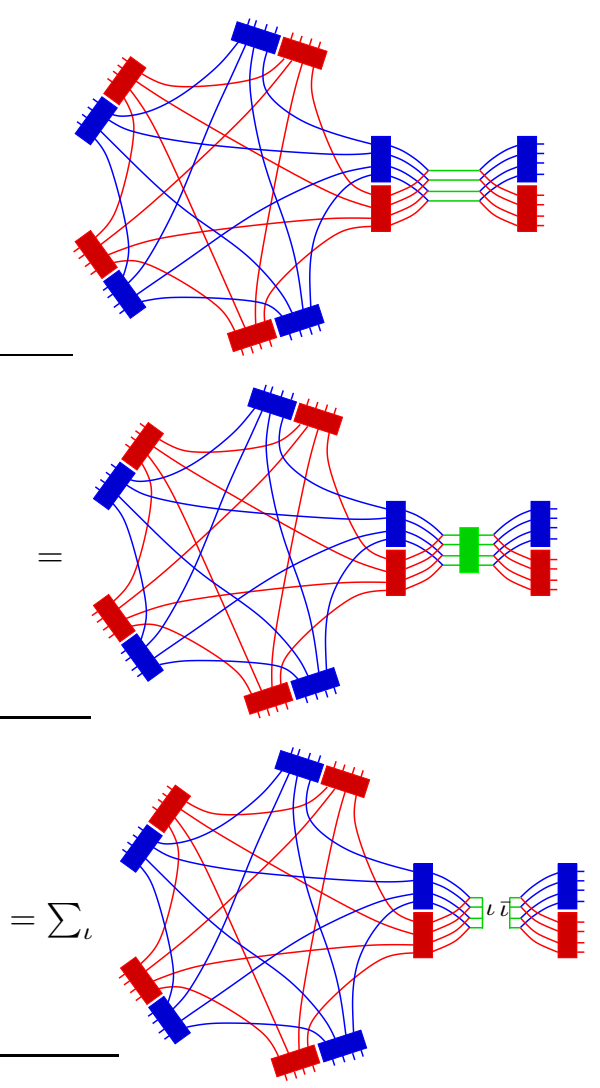

$(62)$

which follows, basically, from the invariance of the Haar measure (9) (in the last line, we have used (17)). More precisely, the integration of the subgroup $S U(2) \in \operatorname{Spin}(4)$, represented by the green box on the right, can be absorbed by suitable redefinition of the integration on the right and left copies of $S U(2)$, represented by the red and blue boxes, respectively. With this, we can already write the spin foam representation of the EPRL model, namely

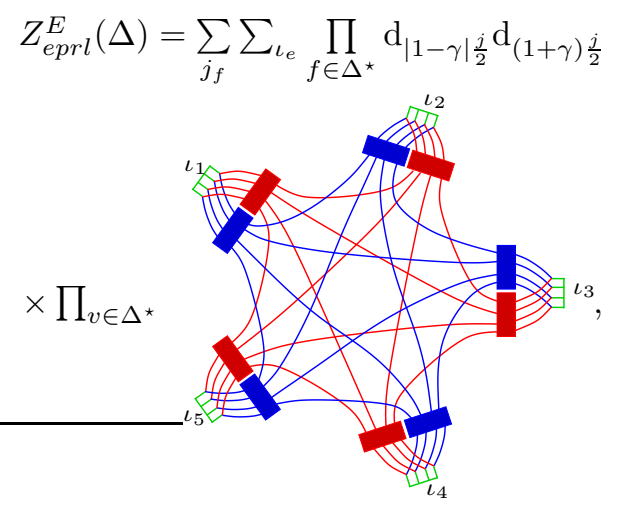

where the vertex amplitude (graphically represented) depends on the 10 spins $j$ associated to the face-wires and the 5 intertwiners associated to the five edges (tetrahedra). As in previous equations, we have left the spin labels of wires implicit for notational simplicity. We can write the previous spin foam amplitude in another form by integrating out all the projectors (boxes) explicitly. Using (17), we get

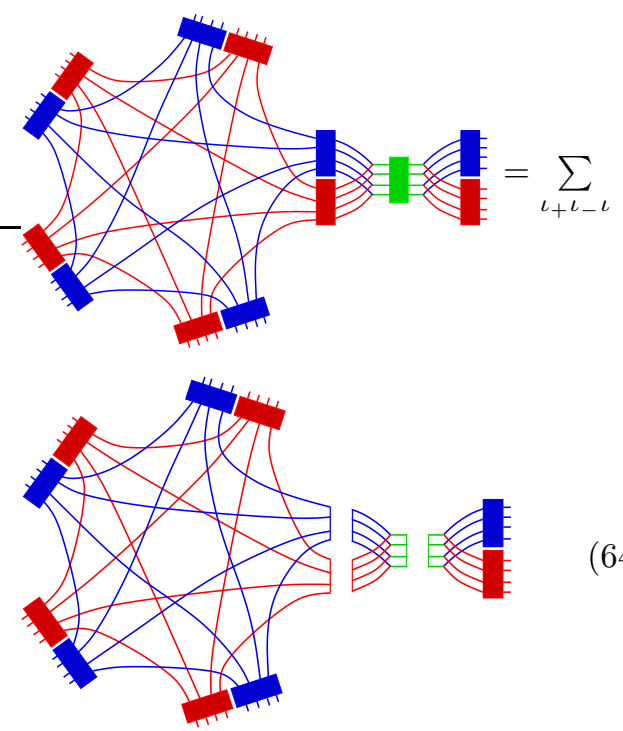

thus replacing this in (61), we get 


$$
Z_{e p r l}^{E}(\Delta)=\sum_{j_{f}} \prod_{f \in \Delta^{\star}} \mathrm{d}_{|\gamma-1| \frac{j}{2}} \mathrm{~d}_{(\gamma+1) \frac{j}{2}} \sum_{\iota_{e}} \prod_{v \in \Delta^{\star}}
$$$$
\sum_{\iota_{1}^{-} \ldots \iota_{5}^{-}} \sum_{\iota_{1}^{+} \ldots \iota_{5}^{+}} \prod_{a=1}^{5} f_{\iota_{a}^{-}, \iota_{a}^{+}}^{\iota_{a}}
$$

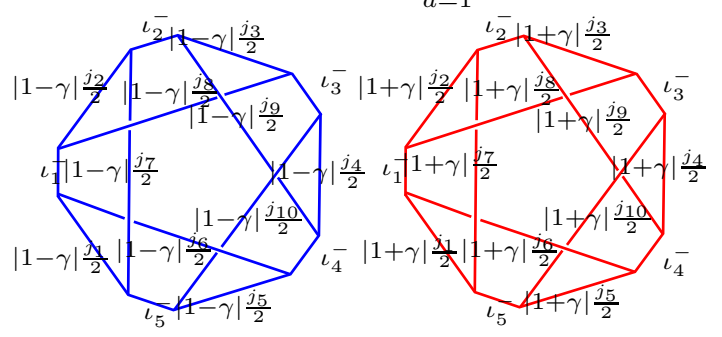

where the coefficients $f_{\iota^{+} \iota^{-}}$are the so-called fusion coefficients which already appear in their graphical form in (64), more explicitly

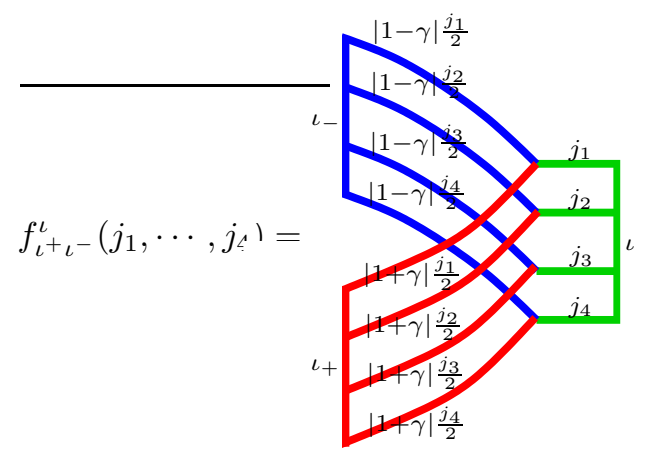

The previous Eq. (66) is the form of the EPRL model as derived in Ref. [5].

\section{iv. Proof of validity of the Plebanski con- straints}

In this section, we prove that the quadratic constraints are satisfied in the sense that their path integral expectation value and fluctuation vanish in the appropriate semiclassical limit.

\section{a. The quadratic Plebanski constraints}

The quadratic Plebanski constraints are

$$
\epsilon_{I J K L} B_{\mu \nu}^{I J} B_{\rho \sigma}^{K L}-e \epsilon_{\mu \nu \rho \sigma} \approx 0 .
$$

The constraints in this form are more suitable for the translation into the discrete formulation. More precisely, according to (6), the smooth fields $B_{\mu \nu}^{I J}$ are now associated with the discrete quantities $B_{\text {triangles }}^{I J}$, or equivalently $B_{f}^{I J}$ as faces $f \in \Delta^{\star}$ are in one-to-one correspondence to triangles in four dimensions. The constraints (67) are local constraints valid at every spacetime point. In the discrete setting, spacetime points are represented by four-simplexes or (more addapted to our discussion) vertices $v \in \Delta^{\star}$. With this, the constraints (67) are discretized as follows:

$$
\begin{array}{r}
\text { Triangle (or diagonal) constraints: } \\
\epsilon_{I J K L} B_{f}^{I J} B_{f}^{K L}=0,
\end{array}
$$

for all $f \in v$, i.e., for each and every face of the 10 possible faces touching the vertex $v$.

$$
\begin{array}{r}
\text { Tetrahedron constraints: } \\
\epsilon_{I J K L} B_{f}^{I J} B_{f^{\prime}}^{K L}=0,
\end{array}
$$

for all $f, f^{\prime} \in v$, so that they are dual to triangles sharing a one-simplex, i.e., belonging to the same tetrahedron out of the five possible ones.

$$
\begin{array}{r}
\text { 4-simplex constraints: } \\
\epsilon_{I J K L} B_{f}^{I J} B_{\bar{f}}^{K L}=e_{v},
\end{array}
$$

for any pair of faces $f, \bar{f} \in v$ that are dual to triangles sharing a single point. The last constraint will require a more detailed discussion. At this point, let us point out that the constraint (70) is interpreted as a definition of the four volume $e_{v}$ of the four-simplex. The constraint requires such definition to be consistent, i.e., the true condition is

$$
\begin{gathered}
\epsilon_{I J K L} B_{f}^{I J} B_{\bar{f}}^{K L}=\epsilon_{I J K L} B_{f^{\prime}}^{I J} B_{\bar{f}^{\prime}}^{K L} \\
=\epsilon_{I J K L} B_{f^{\prime \prime}}^{I J} B_{\bar{f}^{\prime \prime}}^{K L}=\cdots=e_{v}
\end{gathered}
$$

for all five different possible pairs of $f$ and $\bar{f}$ in a four simplex, and where we assume the pairs $f-\bar{f}$ are ordered in agreement with the orientation of the complex $\Delta^{\star}$.

b. The path integral expectation value of the Plebanski constraints

Here we prove that the Plebanski constraints are satisfied by the EPRL amplitudes in the path integral expectation value sense. 
Papers in Physics, vol. 4, ART. 040004 (2012) / A. Perez

The triangle constraints:

We start from the simplest case: The triangle (or diagonal) constraints (68). We choose a face $f \in v$ (dual to a triangle) in the cable-wire-diagram of Eq. (61). This amounts to choosing a pair of wires (right and left representations) connecting two nodes in the vertex cable wire diagram. The two nodes are dual to the two tetrahedra - in the four simplex dual to the vertex - sharing the chosen triangle. Equation (36) shows that

$$
\begin{aligned}
& \epsilon_{I J K L} B_{f}^{I J} B_{f}^{K L} \\
& \quad \propto(1+\gamma)^{2} \quad J_{f}^{-} \cdot J_{f}^{-}-(1-\gamma)^{2} J_{f}^{+} \cdot J_{f}^{+},
\end{aligned}
$$

where $J_{f}^{ \pm}$denotes the self-dual and anti-self-dual parts of $\Pi_{f}^{I J}$. The path integral expectation value of the triangle constraint is then

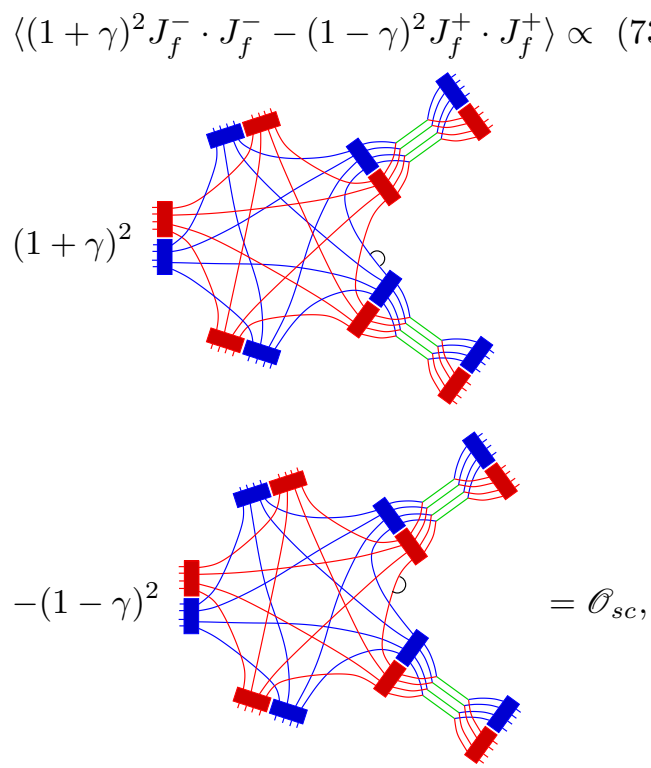

where the double graspings on the anti-self-dual (blue) wire and the self-dual (red) wire represent the action of the Casimirs $J_{f}^{-} \cdot J_{f}^{-}$and $J_{f}^{+} \cdot J_{f}^{+}$, on the cable-wire diagram of the corresponding vertex. Direct evaluation shows that the previous diagram is proportional to $\hbar^{2} j_{f}$ which vanishes in the semiclassical limit $\hbar \rightarrow 0, j \rightarrow \infty$ with $\hbar j=$ constant. We use the notation already adopted in (54) and call such quantity $\mathscr{O}_{s c}$. This proves that the triangle Plebanski constraints are satisfied in the semiclassical sense.
The tetrahedra constraints:

The proof of the validity of the tetrahedra constraints (69). In this case we also have

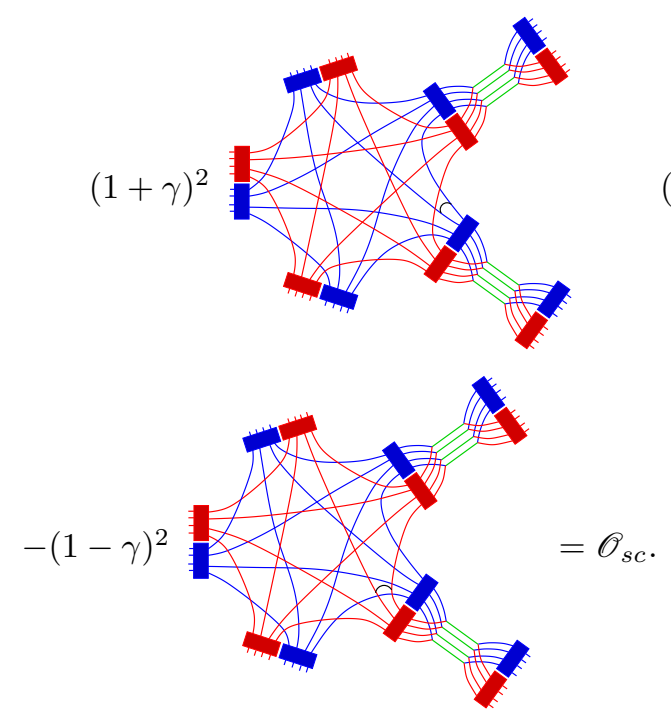

where we have chosen an arbitrary pair of faces. In order to prove this, let us develop the term on the right. The result follows from

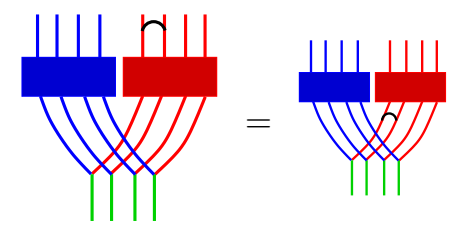

$$
\begin{aligned}
& =\frac{(1+\gamma)}{|1-\gamma|}+\frac{(1+\gamma)^{2}}{(1-\gamma)^{2}}+\mathscr{O}_{s c} \\
& =\frac{(1+\gamma)^{2}}{(1-\gamma)^{2}}
\end{aligned}
$$

where in the first line we have used the fact that the double grasping can be shifted through the group integration (due to gauge invariance (9)). 
In the first and second terms on the second line, we have used Eq. (55) to move the graspings on self-dual wires to the corresponding anti-self-dual wires. Equation (75) immediately follows the previous one; the argument works in the same way for any other pair of faces. Notice that the first equality in Eq. (75) implies that we can view the Plebanski constraint as applied in the frame of the tetrahedron as well as in a Lorentz invariant framework (the double grasping defines an intertwiner operator commuting with the projection $P_{i n v}^{e}$ represented by the box). An analogous statement also holds for the triangle constraints (73).

The 4-simplex constraints

Now we show the validity of the four simplex constraints in their form (71). As we will show below, this last set of constraints follow from the $\operatorname{Spin}(4)$ gauge invariance of the EPRL node (i.e., the validity of the Gauss law) plus the validity of the tetrahedra constraints (69). Gauge invariance of the node takes the following form in graphical notation:

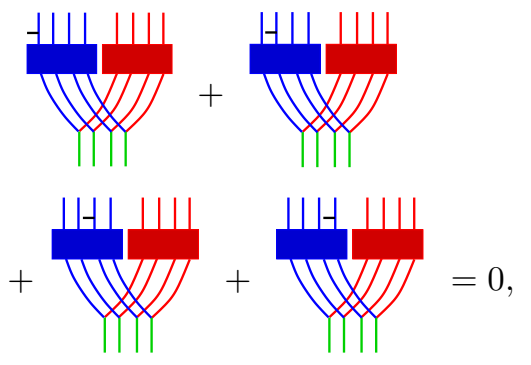

where the above equation represents the gauge invariance under infinitesimal left $S U(2)$ rotations. An analogous equation with insertions on the right is also valid. The validity of the previous equation can, again, be related to the invariance of the Haar measure used in the integration on the gauge group that defines the boxes (9).

Now we choose an arbitrary pair $f$ and $\bar{f}$ (where $\bar{f}$ is one of the three possible faces whose dual triangle only shares a point with the one corresponding to $f$ ) and will show how the four volumen $e_{v}$ defined by it equals the one defined by any other admissible pair. The first step is to show that we get the same result using the pairs $f-\bar{f}$ and $f-\overline{\bar{f}}$, where $\overline{\bar{f}}$ is another of the three admissible faces opposite to $f$. The full result follows from applying the same procedure iteratively to reach any admissible pair. It will be obvious from the treatment given below, that this is possible. Thus, for a given pair of admissible faces, we have 


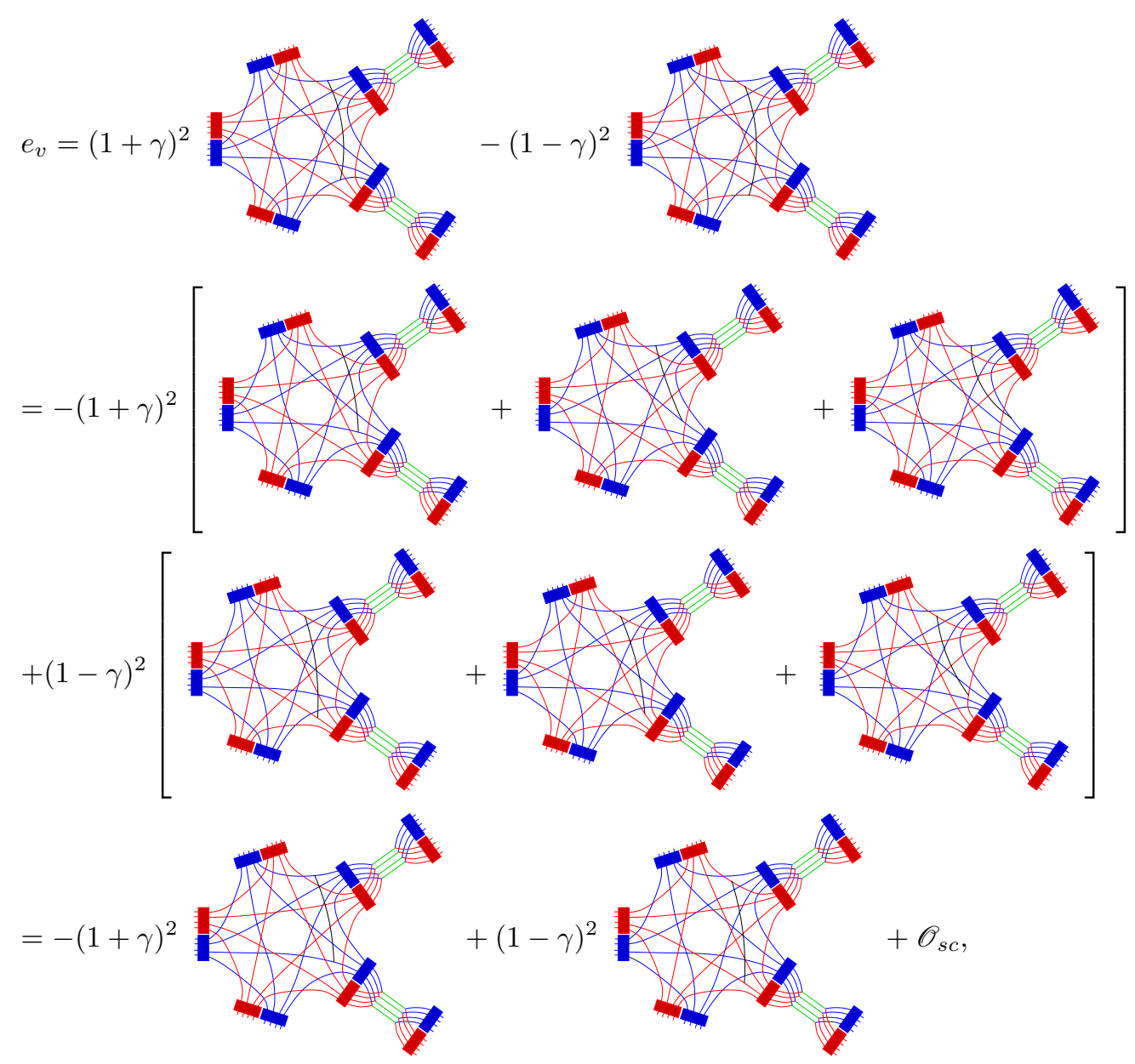

where going from the first line to the second and third lines we have simply used (76) on the bottom graspings on the right and left wires. The last line results from the validity of (69). Notice that the second terms in the second and third lines add up to $\mathscr{O}_{s c}$, as well as the third terms in the second and third line. There is an overall minus sign which amounts for an orientation factor. It should be clear that we can apply the same procedure to arrive at any admissible pair.

\section{c. $P_{\text {eprl }}$ is not a projector}

We will study in detail the object $P_{e p r l}^{e}\left(j_{1}, \cdots, j_{4}\right)$. We see that it is made of two ingredients. The first one is the projection to the maximum weight subspace $\mathscr{H}_{j}$ for $\gamma>1$ in the decomposition of $\mathscr{H}_{j^{+}, j^{-}}$for $j^{ \pm}=(1 \pm \gamma) j / 2\left(j^{ \pm}=(\gamma \pm 1) j / 2\right.$ for $\gamma>1)$ in terms of irreducible representations of an arbitrarily chosen $S U(2)$ subgroup of $\operatorname{Spin}(4)$. The second ingredient is to eliminate the dependence on the choice of subgroup by group averaging with respect to the full gauge group Spin(4). This is diagrammatically represented in (60). However, $P_{e p r l}^{e}\left(j_{1}, \cdots, j_{4}\right)$ is not a projector, namely

$$
P_{e p r l}^{e}\left(j_{1}, \cdots, j_{4}\right)^{2} \neq P_{e p r l}^{e}\left(j_{1}, \cdots, j_{4}\right) .
$$

Technically, this follows from (59) and the fact that

$$
\left[P_{i n v}^{e}\left(\rho_{1} \cdots \rho_{4}\right),\left(\mathscr{Y}_{j_{1}} \otimes \cdots \otimes \mathscr{Y}_{j_{4}}\right)\right] \neq 0
$$

i.e., the projection imposing the linear constraints (defined on the frame of a tetrahedron or edge) 
and the $\operatorname{Spin}(4)$ (or Lorentz) group averagingrendering the result gauge invariant - do not commute. The fact that the $P_{e p r l}^{e}\left(j_{1}, \cdots, j_{4}\right)$ is not a projection operator has important consequences in the mathematical structure of the model:

1. From (61) one can immediately obtain the following expression for the EPRL amplitude

$$
\begin{array}{r}
Z_{\text {eprl }}(\Delta)=\sum_{\rho_{f} \in \mathscr{K}} \prod_{f \in \Delta^{\star}} \mathrm{d}_{|1-\gamma| \frac{j}{2}} \mathrm{~d}_{(1+\gamma) \frac{j}{2}} \\
\times \prod_{e} P_{e p r l}^{e}\left(j_{1}, \cdots, j_{4}\right) .
\end{array}
$$

This expression has the formal structure of expression (13) for BF theory. The formal similarity, however, is broken by the fact that $P_{e p r l}^{e}\left(j_{1}, \cdots, j_{4}\right)$ is not a projection operator. From the formal perspective, there is the possibility for the amplitudes to be defined in terms of a network of projectors (as in BF theory). This might provide an interesting structure that might be of relevance in the definition of a discretization independent model. On the contrary, the failure of $P_{e p r l}^{e}\left(j_{1}, \cdots, j_{4}\right)$ to be a projector may lead, in my opinion, to difficulties in the limit where the complex $\Delta$ is refined: The increasing of the number of edges might produce either trivial or divergent amplitudes $^{2}$.

2. Another difficulty associated with $P_{e p r l}^{e}\left(j_{1}, \cdots, j_{4}\right)^{2} \neq P_{e p r l}^{e}\left(j_{1}, \cdots, j_{4}\right)$ is the failure of the amplitudes of the EPRL model, as defined here, to be consistent with the abstract notion of spin foams as defined in [74]. This is a point of crucial importance under current discussion in the community. The point is that the cellular decomposition $\Delta$ has no physical meaning and is to be interpreted as a subsidiary regulating structure to be removed when computing physical quantities. Spin foams configurations can fit in different ways on a given $\Delta$, yet any of these different embeddings represent the same physical process (like the same gravitational field in different coordinates). Consistency requires the spin

foam amplitudes to be independent of the embedding, i.e., well-defined on the equivalence classes of spin foams as defined by Baez in Ref. [74] (the importance of these consistency requirements was emphasized in Ref. [75]). The amplitude (80) fails this requirement due to $P_{e p r l}^{e}\left(j_{1}, \cdots, j_{4}\right)^{2} \neq P_{e p r l}^{e}\left(j_{1}, \cdots, j_{4}\right)$.

\section{d. The Warsaw proposal}

If one sees the above as difficulties, then there is a simple solution, at least in the Riemannian case. As proposed in Ref. [76,77], one can obtain a consistent modification of the EPRL model by replacing $P_{e p r l}^{e}$ in (80) by a genuine projector $P_{w}^{e}$, graphically

$$
P_{w}^{e}\left(j_{1} \cdots j_{4}\right)=\sum_{\alpha \beta}
$$

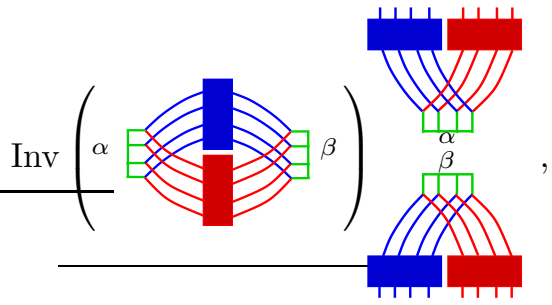

It is easy to check that by construction

$$
\left(P_{w}^{e}\left(j_{1} \cdots j_{4}\right)\right)^{2}=P_{w}^{e}\left(j_{1} \cdots j_{4}\right)
$$

The variant of the EPRL model proposed in Refs. $[76,77]$ takes then the form

$$
\begin{aligned}
& Z_{e p r l}(\Delta)=\sum_{j_{f}} \prod_{f \in \Delta^{\star}} \mathrm{d}_{|1-\gamma| \frac{j}{2}} \mathrm{~d}_{(1+\gamma) \frac{j}{2}} \\
& \times \prod_{e} P_{w}^{e}\left(j_{1}, \cdots, j_{4}\right) \\
& =\sum_{j_{f}} \sum_{\iota_{e v}} \prod_{f \in \Delta^{\star}} \mathrm{d}_{|1-\gamma| \frac{j}{2}} \mathrm{~d}_{(1+\gamma) \frac{j}{2}} \\
& \times \prod_{e \in \Delta^{\star}} g_{\iota_{v_{s}}^{e} \iota_{v_{t}}^{e}} \prod_{v \in \Delta^{\star}}
\end{aligned}
$$

\footnotetext{
${ }^{2}$ This is obviously not clear from the form of (80). We are extrapolating the properties of $\left(P_{e p r l}^{e}\right)^{N}$ for large $N$ to those of the amplitude (80) in the large number of edges limit implied by the continuum limit.
} 
Thus, in the modified EPRL model, edges $e \in \Delta^{\star}$ are assigned pairs of intertwiner quantum numbers $\iota_{v_{s}}^{e}$ and $\iota_{v_{t}}^{e}$ and an edge amplitude given by the matrix elements $g_{\iota_{v_{s}}^{e}, \iota_{v_{t}}^{e}}^{e}$ (where $v_{s}$ and $v_{t}$ stand for the source and target vertices of the given oriented edge). The fact that edges are not assigned a single quantum number is not really significative; one could go to a basis of normalized eigenstates of $P_{w}^{e}$ and rewrite the modified model above as a spin foam model where edges are assigned a single (basis element) quantum number. As the nature of such basis and the quantum geometric interpretation of its elements are not clear at this stage, it seems simpler to represent the amplitudes of the modified model in the above form.

The advantages of the modified model are important. However, a generalization of the above modification of the EPRL model in the Lorentzian case is still lacking. Notice that this modification does not interfere with the results on the semiclassical limit (to leading order), as reviewed in Section VII. The reason for this is that the matrix elements $g_{\alpha \beta}^{e} \rightarrow \delta_{\alpha \beta}$ in that limit [78].

\section{v. The coherent states representation}

We have written the amplitude defining the EPRL model by constraining the state sum of BF theory. For semiclassical studies that we will review in Section VII, it is convenient to express the EPRL amplitude in terms of the coherent states basis. The importance of coherent states in spin foam models was put forward in Ref. [49] and explicitly used to re-derive the EPRL model in Ref. [79]. The coherent state technology was used by Freidel and Krasnov in [6] to introduce a new kind of spin foam models for gravity: The FK models. In some cases, the FK model is equivalent to the EPRL model. We will review this in detail in Section IV.

The coherent state representation of the EPRL model is obtained by replacing (27) in each of the intermediate $S U(2)$ (green) wires in the expression (61) of the EPRL amplitudes, namely

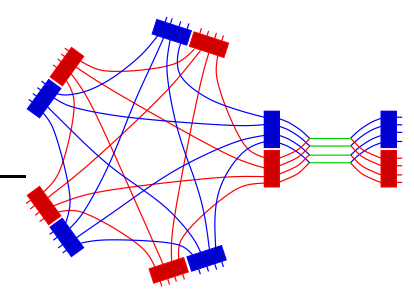

The case $\gamma<1$

In this case, the coherent state property (28) implies
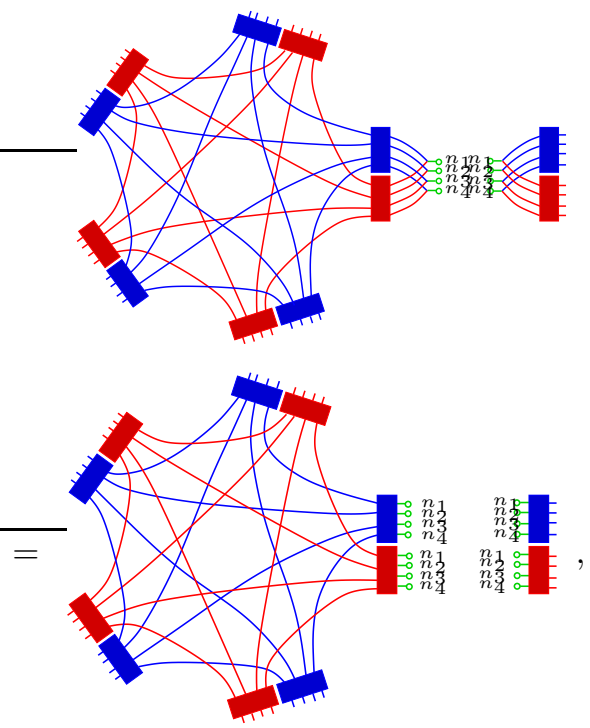

where we have used, in the last line, the fact that for $\gamma<1$ the representations $j$ of the subgroup $S U(2) \in \operatorname{Spin}(4)$ are maximum weight, i.e., $j=j^{+}+j^{-}$. Doing this at each edge, we get 
where the discrete action

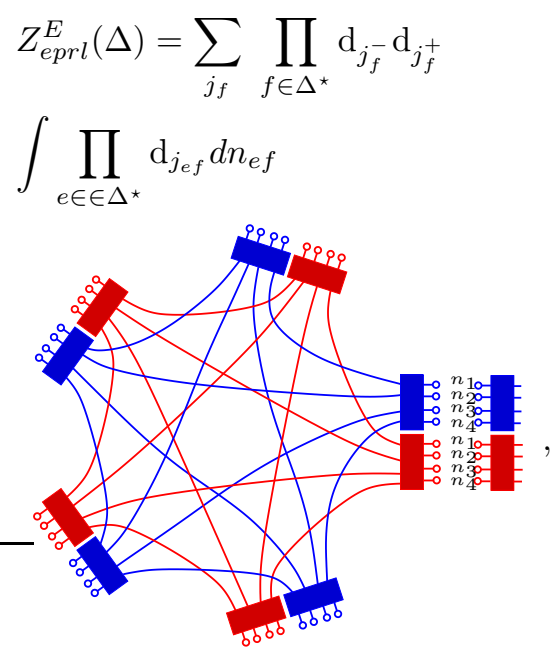

(86)

where we have explicitly written the $n \in S^{2}$ integration variables on a single cable. The expression above is very similar to the coherent states representation of $\operatorname{Spin}(4)$ BF theory given in Eq. (29). In fact, one gets the above expression if one starts from the expression (29) and sets $n_{\text {ef }}^{+}=n_{\text {ef }}^{-}=n_{e f}$ while dropping, for example, all the sphere integrations corresponding to the $n_{e f}^{+}$(or equivalently $\left.n_{e f}^{-}\right)$. Moreover, by construction, the coherent states participating in the previous amplitude satisfy the linear constraints (45) in expectation values, namely

$$
\begin{aligned}
& \left\langle j, n_{e f}\left|D_{f}^{i}\right| j, n_{e f}\right\rangle \\
& =\left\langle j, n_{e f}\left|(1-\gamma) J_{f}^{+i}+(1+\gamma) J_{f}^{-i}\right| j, n_{e f}\right\rangle \\
& =0
\end{aligned}
$$

Thus, the coherent states participating in the above representation of the EPRL amplitudes solve the linear simplicity constraints in the usual semiclassical sense. The same manipulations leading to (89) in Section II lead to a discrete effective action for the EPRL model, namely

$$
\begin{aligned}
& Z_{e p r l}^{\gamma<1}=\sum_{j_{f}} \prod_{f \in \Delta^{\star}} \mathrm{d}_{(1-\gamma) \frac{j_{f}}{2}} \mathrm{~d}_{(1+\gamma) \frac{j_{f}}{2}} \\
& \times \int \prod_{e \in \Delta^{\star}} \mathrm{d}_{j_{e f}} d n_{e f} d g_{e f}^{-} d g_{e f}^{+} \exp \left(S_{j^{ \pm}, \mathbf{n}}^{\gamma<1}\left[g^{ \pm}\right]\right),
\end{aligned}
$$

040004-21

040004-21

$$
\begin{aligned}
& S_{j^{ \pm}, \mathbf{n}}^{\gamma<1}\left[g^{ \pm}\right] \\
& =\sum_{v \in \Delta^{\star}}\left(S_{(1-\gamma) \frac{j_{f}}{2}, \mathbf{n}}^{v}\left[g^{-}\right]+S_{(1+\gamma) \frac{j_{f}}{2}, \mathbf{n}}^{v}\left[g^{+}\right]\right)
\end{aligned}
$$

with

$$
\begin{aligned}
& S_{j, \mathbf{n}}^{v}[g] \\
& =\sum_{a<b=1}^{5} 2 j_{a b} \ln \left\langle n_{a b}\left|g_{a}^{-1} g_{b}\right| n_{b a}\right\rangle,
\end{aligned}
$$

and the indices $a, b$ label the five edges of a given vertex. The previous expression is exactly equal to the form (11) of the BF amplitude. In the case of the gravity models presented here, the coherent state path integral representation (analogous to (31)) will be the basic tool for the study of the semiclassical limit of the models and the relationship with Regge discrete formulation of general relativity.

The case $\gamma>1$

The case $\gamma>1$ is more complicated [80]. The reason for this is that the step (85), directly leading to the discrete action in the previous case, is no longer valid, as the representations of the subgroup $S U(2) \in \operatorname{Spin}(4)$ are now minimum instead of maximum weight. However, the representations $j^{+}=j^{-}+j$ are maximum weight. We can, therefore, insert coherent states resolution of the identity on the right representations and get: 
PApers in Physics, vol. 4, ART. 040004 (2012) / A. Perez

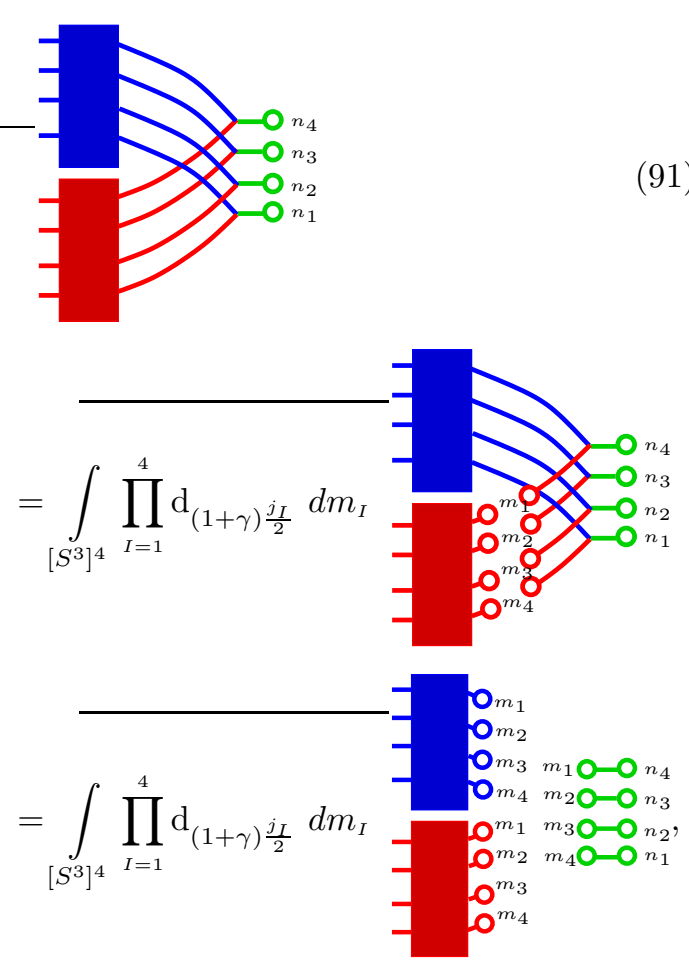

where we are representing the relevant part of the diagram appearing in Eq. (85). In the last line, we have used $j^{+}=j+j^{-}$(i.e., maximum weight), and the graphical notation $m \circ-\circ n \equiv\langle m \mid n\rangle$ as it follows from our previous conventions. With this, one gets

$$
\begin{aligned}
& Z_{e p r l}^{\gamma>1}= \\
& \sum_{j_{f}} \prod_{f \in \Delta^{\star}} \mathrm{d}_{(1-\gamma) \frac{j_{f}}{2}} \mathrm{~d}_{(1+\gamma) \frac{j_{f}}{2}} \\
& \times \int \prod_{e \in \Delta^{\star}} \mathrm{d}_{j_{e f}} \mathrm{~d}_{(1+\gamma) \frac{j_{e f}}{2}} d n_{e f} d m_{e f} d g_{e f}^{-} d g_{e f}^{+} \\
& \times \exp \left(S_{j^{ \pm}, \mathbf{n}, \mathbf{m}}^{\gamma>1}\left[g^{ \pm}\right]\right),
\end{aligned}
$$

where the discrete action

$$
S_{j^{ \pm}, \mathbf{n}, \mathbf{m}}^{\gamma>1}\left[g^{ \pm}\right]=\sum_{v \in \Delta^{\star}} S_{j^{ \pm}, \mathbf{n}, \mathbf{m}}^{v}\left[g^{ \pm}\right]
$$

with

$$
\begin{aligned}
& S_{j^{ \pm}, \mathbf{n}, \mathbf{m}}^{v}\left[g^{ \pm}\right] \\
& =\sum_{1 \leq a<b \leq 5}\left[j_{a b}(1+\gamma) \log \left(\left\langle m_{a b}\left|g_{a b}^{+}\right| m_{b a}\right\rangle\right)\right. \\
& +j_{a b}(\gamma-1) \log \left(\left\langle m_{a b}\left|g_{a b}^{-}\right| m_{b a}\right\rangle\right) \\
& \left.+2 j_{a b}\left(\log \left(\left\langle n_{a b} \mid m_{a b}\right\rangle\right)+\log \left(\left\langle m_{b a} \mid n_{b a}\right\rangle\right)\right)\right] .
\end{aligned}
$$

\section{a. Some additional remarks}

It is important to point out that the commutation relations of basic fields - reflecting the simple algebraic structure of $\operatorname{spin}(4)$ - used here are the ones induced by the canonical analysis of $\mathrm{BF}$ theory presented previously. The presence of constraints generally modifies canonical commutation relations, in particular in the presence of second class constraints. For some investigation of the issue in the context of the EPRL and FK models, see Ref. [69]. In Ref. [81], it is pointed out that the presence of secondary constraints in the canonical analysis of Plebanski action should be translated into additional constraints in the holonomies of the spin foam models here considered (see also Ref. [82]). A possible view is that the simplicity constraints are here imposed for all times and thus secondary constraints should be imposed automatically.

There are alternative derivations of the models presented in the previous sections. In particular, one can derive them from a strict Lagrangean approach of Plebanski's action. Such viewpoint is taken in Refs. [83-85]. The path integral formulation of Plebansky theory using commuting $B$-fields was studied in Ref. [86], where it is shown that only in the appropriate semiclassical limit the amplitudes coincide with the ones presented in the previous sections (this is just another indication that the construction of the models has a certain semiclassical input; see below). The spin foam quantization of the Holst formulation of gravity via cubulations was investigated in Ref. [87]. The simplicity constraints can also be studied from the perspective of the $U(N)$ formulation of quantum geometry [88]. Such $U(N)$ treatment is related to previous work $[89,90]$ which has been extended to a completely new perspective on quantum geometry with possible advantageous features [91,92]. For additional discussion on the simplicity constraints, see Ref. 
[93].

\section{vi. Presentation of the EPRL Lorentzian model}

As it was briefly discussed in Section III, unitary irreducible representations of $S L(2, \mathbb{C})$ are infinite dimensional and labeled by a positive real number $p \in \mathbb{R}^{+}$and a half-integer $k \in \mathbb{N} / 2$. These representations are the ones that intervene in the harmonic analysis of square integrable functions of $S L(2, \mathbb{C})$ [64]. Consequently, one has an explicit expression of the delta function distribution (defined on such test function), namely

$$
\delta(g)=\sum_{k} \int_{\mathbb{R}^{+}} d p\left(p^{2}+k^{2}\right) \sum_{j, m} D_{j m j m}^{p, k}(g)
$$

where $D_{j m j^{\prime} m^{\prime}}^{p, k}(g)$ with $j \geq k$ and $j \geq m \geq-j$ (similarly for the primed indices) are the matrix elements of the unitary representations $p-k$ in the so-called canonical basis [63]. One can use the previous expression, the Lorentzian version of Eq. (11), in order to introduce a formal definition of the BF amplitudes, which now would involve integration of the continuous labels $p_{f}$, in addition to sums over discrete quantum numbers such as $k, j$ and $m$. The Lorentzian version of the EPRL model can be obtained from the imposition of the linear simplicity constraints to this formal expression. As the continuum labels $p_{f}$ are restricted to $p_{f}=\gamma j_{f}$, the Lorentzian EPRL model becomes a state-sum model as its Riemannian relative. Using the following graphical notation

$$
D_{j m j^{\prime} m^{\prime}}^{p, k}(g)=j, m \frac{p}{k} j^{\prime}, m^{\prime}
$$

the amplitude is

$$
=\sum_{j_{f}} \prod_{f \in \Delta^{\star}}^{Z_{e p r l}^{L}(\Delta)}\left(1+\gamma^{2}\right) j_{f}^{2}
$$

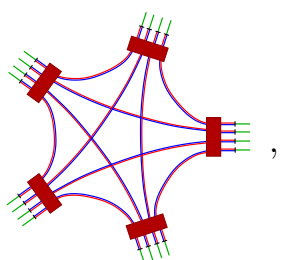

where the boxes now represent $S L(2, \mathbb{C})$ integrations with the invariant measure. The previous amplitude is equivalent to its spin foam representation

$$
Z_{e p r l}^{L}(\Delta)=\sum_{j_{f}} \sum_{\iota_{e}} \prod_{f \in \Delta^{\star}}\left(1+\gamma^{2}\right) j_{f}^{2}
$$

The vertex amplitude is divergent due to the presence of a redundant integration over $S L(2, \mathbb{C})$. It becomes finite by dropping an arbitrary integration, i.e., removing any of the 5 boxes in the vertex expression [94].

\section{a. The coherent state representation}

It is immediate to obtain the coherent states representation of the Lorentzian models. As in the Riemannian case, one simply inserts resolution of the identities (22) on the intermediate $S U(2)$ (green) wires in (97), from where it results

$$
Z_{e p r l}^{L}(\Delta)=\sum_{j_{f}} \prod_{f \in \Delta^{\star}}\left(1+\gamma^{2}\right) j^{2}
$$

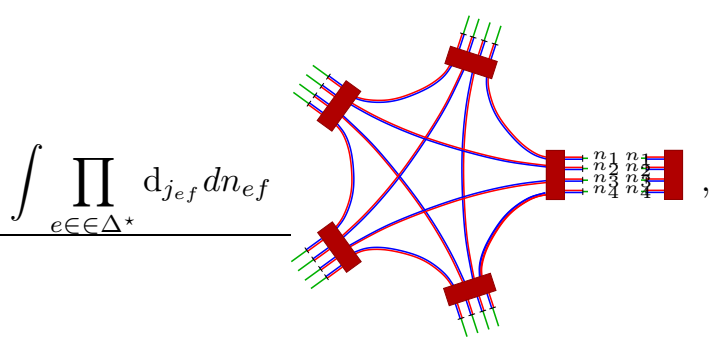

\section{The Freidel-Krasnov model}

Shortly after the appearance of the paper in Ref. [4], Freidel and Krasnov [6] introduced a set of new spin foam models for four-dimensional gravity using the coherent state basis of the quantum tetrahedron of Livine and Speziale [49]. The idea is to 
Papers in Physics, vol. 4, Art. 040004 (2012) / A. Perez

impose the linearized simplicity constraints (45) directly as a semiclassical condition on the coherent state basis. As we have seen above, coherent states are quantum states of the right and left tetrahedra in BF theory which have a clear-cut semiclassical interpretation through their property (26). We have also seen that the imposition of the linear constraints (45) a la EPRL is in essence semiclassical as they are strictly valid only in the large spin limit. In the FK approach one simply accepts from the starting point that, due to their property of a non-defining set that is closed under commutation relations, the Plebansky constraints are to be imposed semiclassically. One defines new models by restricting the set of coherent states entering in the coherent state representation of $\operatorname{Spin}(4)$ BF theory (29) to those that satisfy condition (45) in expectation values. They also emphasize how the model [4] corresponds, indeed, to the sector $\gamma=\infty$ which has been shown to be topological [95].

\section{The case $\gamma<1$}

For $\gamma<1$, the vertex amplitude is identical to the EPRL model. This is apparent in the coherent state expression of the EPRL model (88). Thus, we have

$$
\begin{aligned}
& Z_{f k}^{\gamma<1}(\Delta)=\sum_{j_{f}} \prod_{f \in \Delta^{\star}} \mathrm{d}_{|1-\gamma| \frac{j}{2}} \mathrm{~d}_{(1+\gamma) \frac{j}{2}} \\
& \prod_{e \in \Delta^{\star}} \int \mathrm{d}_{(1+\gamma) \frac{j}{2}} \mathrm{~d}_{(\gamma-1) \frac{j_{e f}}{2}} d n_{e f}
\end{aligned}
$$

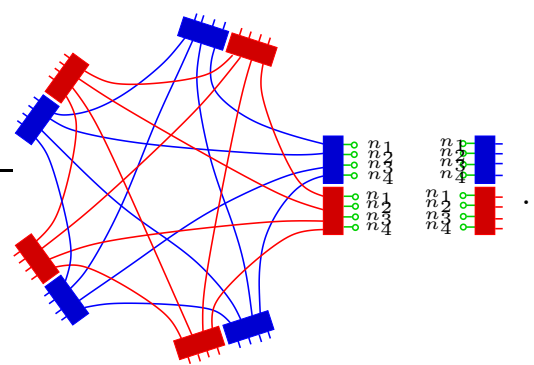

From the previous expression, we conclude that the vertex amplitudes of the FK and EPRL model coincide for $\gamma<1$

$$
A_{v f k}^{\gamma<1}=A_{v e p r l}^{\gamma<1} .
$$

Notice, however, that different weights are assigned to edges in the FK model. This is due to the fact that one is restricting the $\operatorname{Spin}(4)$ resolution of identity in the coherent basis in the previous expression, while in the EPRL model the coherent state resolution of the identity is used for $S U(2)$ representations. This difference is important and has to do with the still unsettled discussion concerning the measure in the path integral representation.

\section{The case $\gamma>1$}

For the case $\gamma>1$, the FK amplitude is given by

$$
\begin{aligned}
& Z_{f k}^{\gamma>1}(\Delta)=\sum_{j_{f}} \prod_{f \in \Delta^{\star}} \mathrm{d}_{|1-\gamma| \frac{j}{2}} \mathrm{~d}_{(1+\gamma) \frac{j}{2}} \\
& \prod_{e \in \Delta^{\star}} \int \mathrm{d}_{(1+\gamma) \frac{j}{2}} \mathrm{~d}_{(\gamma-1) \frac{j_{e f}}{2}} d n_{e f}
\end{aligned}
$$

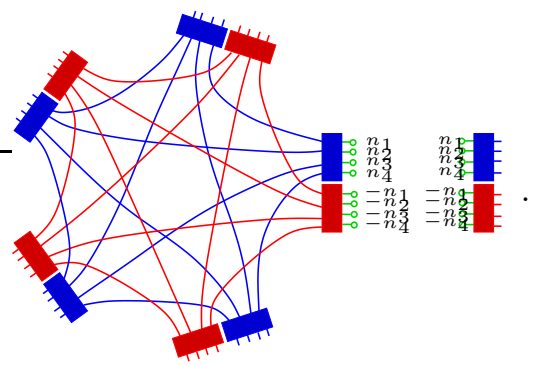

The study of the coherent state representation of the FK model for $\gamma>1$, in comparison with Eq. (92) for the EPRL model, clearly shows the difference between the two models in this regime.

$$
\begin{aligned}
& Z_{f k}^{\gamma}=\sum_{j_{f}} \prod_{f \in \Delta^{\star}} \mathrm{d}_{(1-\gamma) \frac{j_{f}}{2}} \mathrm{~d}_{(1+\gamma) \frac{j_{f}}{2}} \\
& \int \prod_{e \in \Delta^{\star}} \mathrm{d}_{|1-\gamma| \frac{j_{e f}}{2}} \mathrm{~d}_{(1+\gamma) \frac{j_{e f}}{2}} d n_{e f} d g_{e f}^{-} d g_{e f}^{+} \\
& \exp \left(S_{j^{ \pm}, \mathbf{n}}^{f k}\left[g^{ \pm}\right]\right)
\end{aligned}
$$

where the discrete action

$$
\begin{aligned}
S_{j^{ \pm}, \mathbf{n}}^{f k}\left[g^{ \pm}\right]= & \sum_{v \in \Delta^{\star}}\left[S_{(1-\gamma) \frac{j_{f}}{2}, \mathbf{n}}^{v}\left[g^{-}\right]\right. \\
& \left.+S_{(1+\gamma) \frac{j_{f}}{2}, s(\gamma) \mathbf{n}}^{v}\left[g^{+}\right]\right],
\end{aligned}
$$


where $s(\gamma)=\operatorname{sign}(1-\gamma)$ and

$$
S_{j, \mathbf{n}}^{v}[g]=\sum_{a<b=1}^{5} 2 j_{a b} \ln \left\langle n_{a b}\left|g_{a}^{-1} g_{b}\right| n_{b a}\right\rangle,
$$

with the indices $a, b$ labeling the five edges of a given vertex.

\section{Boundary data for the new mod- els and relationship with the canonical theory}

So far, we have considered cellular complexes with no boundaries. Transition amplitudes are expected to be related to the definition of the physical scalar product. In order to define them, one needs to consider complexes with boundaries. Boundary states are defined on the boundary of the dual two-complex $\Delta^{\star}$ that we denote $\partial \Delta^{\star}$. The object $\partial \Delta^{\star}$ is a one-complex (a graph). According to the construction of the model (Section III), boundary states are in one-to-one correspondence with $S U(2)$ spin networks. This comes simply from the fact that links (one-cells) $\ell \in \partial \Delta^{\star}$ inherit the spins labels (unitary irreducible representations of the subgroup $S U(2))$ from the boundary faces while nodes (zero-cells) $n \in \partial \Delta^{\star}$ inherit the intertwiner levels from boundary edges.

At this stage, one can associate the boundary data with elements of a Hilbert space. Being in one-to-one correspondence with $S U(2)$ spin networks, a natural possibility is to associate to them an element of the kinematical Hilbert space of LQG. More precisely, with a given colored boundary graph $\gamma$ with links labeled by spins $j_{\ell}$ and nodes labeled by intertwiners $\iota_{n}$, we associate a cylindrical function $\Psi_{\gamma,\left\{j_{\ell}\right\},\left\{\iota_{n}\right\}} \in \mathscr{L}^{2}\left(S U(2)^{N_{\ell}}\right)$, where here $N_{\ell}$ denotes number of links in the graph $\gamma$. In this way, the boundary Hilbert space associated with $\partial \Delta^{\star}$ is isomorphic (if one used the natural AL measure) with the Hilbert space of LQG truncated to that fixed graph. Moreover, geometric operators, such as volume and area defined in the covariant context, are shown to coincide with the corresponding operators defined in the canonical formulation $[67,96]$. Now, if cellular complexes are dual to triangulations, then the boundary spin networks can have at most four-valent nodes. This limitation can be easily overcome: As in BF theory, the
EPRL amplitudes can be generalized to arbitrary complexes with boundaries given by graphs with nodes of arbitrary valence. The extension of the model to arbitrary complexes has been first studied in Refs. [97,98]. It has also been revisited in Refs. [68].

Alternatively, one can associate the boundary states with elements of $\mathscr{L}^{2}\left(\operatorname{Spin}(4)^{N_{\ell}}\right)$ (in the Riemannian models) - or carefully define the analog of spin network states as distributions in the Lorentzian case (see Refs. [99] for some insights on the problem of defining a gauge invariant Hilbert space of graphs for non-compact gauge groups). In this case, one gets special kinds of spin network states. These are a subclass of the so-called projected spin networks introduced in Refs. [100,101] in order to define a heuristic quantization of the (non-commutative and very complicated) Dirac algebra of a Lorentz connection formulation of the phase space of gravity [100,102-107]. The fact that this special subclass of projected spin networks appears naturally as a boundary state of the new spin foams is shown in Ref. [108].

Due to their similarity to $\gamma<1$, the same relationship between boundary data and elements of the kinematical Hilbert space holds for the FK model. However, such simple relationship does not hold for the model in the case $\gamma>1$.

It is important to mention that the knotting properties of boundary spin networks do not seem to play a role in present definitions of transition amplitudes [109].

\section{Further developments and re- lated models}

The spin foam amplitudes discussed in the previous sections have been introduced by constraining the BF histories through the simplicity constraints. However, in the path integral formulation, the presence of constraints has the additional effect of modifying the weights with which those histories are to be summed: Second class constraints modify the path integral measure (in the spin foam context this issue was raised in Ref. [75]). As pointed out before, this question has not been completely settled in the spin foam community yet. The explicit modification of the formal measure in terms of continuous variables for the Plebansky action was pre- 
sented in Ref. [110]. A systematic investigation of the measure in the spin foam context was attempted in Ref. [111] and [112]. As pointed out in Ref. [75], there are restrictions in the manifold of possibilities coming from the requirement of background independence. The simple BF measure chosen in the presentation of the amplitudes in the previous sections satisfies these requirements. There are other consistent possibilities; see, for instance, Ref. [113] for a modified measure which remains extremely simple and is suggested from the structure of LQG.

An important question is the relationship between the spin foam amplitudes and the canonical operator formulation. The question of whether one can reconstruct the Hamiltonian constraints out of spin foam amplitudes has been analysed in detail in three dimensions. For the study of quantum three-dimensional gravity from the BF perspective, see Ref. [114]. We will, in fact, present this perspective in detail in the three dimensional part of this article. For the relationship with the canonical theory using variables that are natural from the Regge gravity perspective, see $[115,116]$. There are generalizations of Regge variables more adapted to the interpretation of spin foams [117]. In four dimensions, the question has been investigated in Ref. [118] in the context of the new spin foam models. In the context of group field theories, this issue is explored in Ref. [119]. Finally, spin foams can, in principle, be obtained directly from the implementation of the Dirac program using path integral methods. This has been explored in Refs. [120,121], from which a discrete path integral formulation followed [122]. The question of the relationship between covariant and canonical formulations in the discrete setting has been analyzed also in Ref. [123].

By construction, all tetrahedra in the FK and EPRL models are embedded in a spacelike hypersurface and hence have only spacelike triangles. It seems natural to ask the question of whether a more general construction allowing for timelike faces is possible. The models described in previous sections have been generalized in order to include timelike faces in the work of F. Conrady [124-126]. An earlier attempt to define such models in the context of the Barrett-Crane model can be found in Refs. [127].

The issue of the coupling of the new spin foam models to matter remains to a large extend unexplored territory. Nevertheless, some results can be found in the literature. The coupling of the Barrett-Crane model (the $\gamma \rightarrow \infty$ limit of the EPRL model) to Yang-Mills fields was studied in Ref. [128]. More recently, the coupling of the EPRL model to fermions has been investigated in Refs. [129, 130]. A novel possibility of unification of the gravitational and gauge fields was recently proposed in Ref. [131].

The introduction of a cosmological constant in the construction of four-dimensional spin foam models has a long history. Barrett and Crane introduced a vertex amplitude [132], in terms of the Crane and Yetter model [13], for BF theory with cosmological constant. The Lorentzian quantum deformed version of the previous model was studied in Ref. [133]. For the new models, the coupling with a cosmological constant is explored in terms of the quantum deformation of the internal gauge symmetry in Refs. [134,135], as well as (independently) in Ref. [136]. The asymptotics of the vertex amplitude are shown to be consistent with a cosmological constant term in the semiclassical limit in Ref. [137].

The spin foam approach applied to quantum cosmology has been explored in Refs. [138-143]. The spin foam formulation can also be obtained from the canonical picture provided by loop quantum cosmology (see Ref. [144] and references therein). This has been explored systematically in Refs. [145-148].

As we have discussed in the introduction of the new models, Heisenberg uncertainty principle precludes the strong imposition of the Plebanski constraints that reduce BF theory to general relativity. The results of the semiclassical limit of these models seem to indicate that metric gravity should be recovered in the low energy limit. However, it seems likely that the semiclassical limit could be related to certain modifications of Plebanski's formulation of gravity [149-153]. A simple interpretation of the new models in the context of the bi-gravity paradigm proposed in Ref. [154] could be of interest.

As it was already pointed out in Ref. [74], spin foams can be interpreted in close analogy to Feynman diagrams. Standard Feynman graphs are generalized to 2-complexes and the labeling of propagators by momenta to the assignment of spins to 
faces. Finally, momentum conservation at vertices in standard feynmanology is now represented by spin-conservation at edges, ensured by the assignment of the corresponding intertwiners. In spin foam models, the non-trivial content of amplitudes is contained in the vertex amplitude which, in the language of Feynman diagrams, can be interpreted as an interaction. This analogy is indeed realized in the formulation of spin foam models in terms of a group field theory (GFT) $[155,156]$.

The GFT formulation resolves, by definition, the two fundamental conceptual problems of the spin foam approach: Diffeomorphism gauge symmetry and discretization dependence. The difficulties are shifted to the question of the physical role of $\lambda$ and the convergence of the corresponding perturbative series.

This idea has been studied in more detail in three dimensions. In Ref. [157], the scaling properties of the modification of the Boulatov group field theory introduced in Ref. [158] were studied in detail. In a further modification of the previous model (known as colored tensor models [159], new techniques based on a suitable $1 / N$ expansion imply that amplitudes are dominated by spherical topology [160]. Moreover, it seems possible that the continuum limit might be critical as in certain matrix models [161-165]. However, it is not yet clear if there is a sense in which these models correspond to a physical theory. The naive interpretation of the models is that they correspond to a formulation of $3 \mathrm{~d}$ quantum gravity including a dynamical topology.

\section{Results on the semiclassical limit of EPRL-FK models}

Having introduced the relevant spin foam models in the previous sections, we now present the results of the large spin asymptotics of the spin foam amplitudes suggesting that on a fixed discretization the semiclassical limit of the EPRL-FK models is given by Regge's discrete formulation of general relativity $[80,166]$.

The semiclassical limit of spin foams is based on the study of the the large spin limit asymptotic behavior of coherent state spin foam amplitudes. The notion of large spin can be defined by the rescaling of quantum numbers and Planck constant accord- ing to $j \rightarrow \lambda j$ and $\hbar \rightarrow \hbar / \lambda$ and taking $\lambda>>1$. In this limit, the quantum geometry approximates the classical one when tested with suitable states (e.g., coherent states). However, the geometry remains discrete during this limiting process as the limit is taken on a fixed regulating cellular structure. That is why one usually makes a clear distinction between semiclassical limit and the continuum limit. In the semiclassical analysis presented here, one can only hope to make contact with discrete formulations of classical gravity. Hence, the importance of Regge calculus in the discussion of this section.

The key technical ingredient in this analysis is the representation of spin foam amplitudes in terms of the coherent state basis introduced in Subsection i. Here we follow Refs. [80, 166-169]. The idea of using coherent states and discrete effective actions for the study of the large spin asymptotics of spin foam amplitudes was put forward in Refs. $[170,171]$. The study of the large spin asymptotics has a long tradition in the context of quantum gravity, dating back to the study of Ponzano-Regge [26]. More directly related to our discussion, here are the early works $[172,173]$. The key idea is to use asymptotic stationary phase methods for the amplitudes written in terms of the discrete actions presented in the previous section.

In this section, we review the results of the analysis of the large spin asymptotics of the EPRL vertex amplitude for both the Riemannian and Lorentztian models. We follow the notation and terminology of Ref. [80] and related papers.

\section{b. $\mathrm{SU}(2) 15 j$-symbol asymptotics}

As $S U(2)$ BF theory is quite relevant for the construction of the EPRL-FK models, the study of the large spin asymptotics of the $S U(2)$ vertex amplitude is a key ingredient in the analysis of [80]. The coherent state vertex amplitude is

$$
\begin{aligned}
& 15 j(j, \mathbf{n}) \\
& =\int \prod_{a=1}^{5} d g_{a} \prod_{1 \leq a \leq b \leq 5}\left\langle n_{a b}\left|g_{a}^{-1} g_{b}\right| n_{b a}\right\rangle^{2 j_{a b}}
\end{aligned}
$$

which depends on 10 spins $j_{a b}$ and 20 normals $n_{a b} \neq n_{b a}$. The previous amplitude can be expressed as 


$$
\begin{gathered}
15 j(j, \mathbf{n})=\int \prod_{a=1}^{5} d g_{a} \prod_{1 \leq a \leq b \leq 5} \exp S_{j, \mathbf{n}}[g], \\
S_{j, \mathbf{n}}[g]=\sum_{a<b=1}^{5} 2 j_{a b} \ln \left\langle n_{a b}\left|g_{a}^{-1} g_{b}\right| n_{b a}\right\rangle,
\end{gathered}
$$

and the indices $a, b$ label the five edges of a given vertex. The previous expression is equal to the form (11) of the BF amplitude. In the case of the EPRL model studied in Section III, the coherent state representation - see Eqs. 88, 92, and 97is the basic tool for the study of the semiclassical limit of the models and the relationship with Regge discrete formulation of general relativity.

In order to study the asymptotics of (105), one needs to use extended stationary phase methods due to the fact that the action (106) is complex (see Refs. [170,171]). The basic idea is that, in addition to stationarity, one requires real part of the action to be maximal. Points satisfying these two conditions are called critical points. As the real part of the action is negative definite, the action at critical points is purely imaginary.

Notice that the action (106) depends parametrically on 10 spins $j$ and 20 normals $\mathbf{n}$. These parameters define the so-called boundary data for the four simplex $v \in \Delta^{\star}$. Thus, there is an action principle for every given boundary data. The number of critical points and their properties depend on these boundary data, hence the asymptotics of the vertex amplitude is a function of the boundary data. Different cases are studied in detail in Ref. [80]. Here we present their results in the special case where the boundary data describe a non-degenerate Regge geometry for the boundary of a four simplex. These data are referred to as Regge-like, and satisfy the gluing constraints. For such boundary data, the action (106) has exactly two critical points, leading to the asymptotic formula

$$
\begin{aligned}
& 15 j(\lambda j, \mathbf{n}) \sim \frac{1}{\lambda^{12}}\left[N_{+} \exp \left(i \sum_{a<b} \lambda j_{a b} \Theta_{a b}^{E}\right)\right. \\
& \left.+N_{-} \exp \left(-i \sum_{a<b} \lambda j_{a b} \Theta_{a b}^{E}\right)\right]
\end{aligned}
$$

where $\Theta_{a b}$ are the appropriate dihedral angles defined by the four-simplex geometry. Finally, the $N_{ \pm}$are constants that do not scale with $\lambda$.

\section{c. The Riemannian EPRL vertex asymptotics}

The previous result, together with the fact that the EPRL amplitude for $\gamma<1$ is a product of $S U(2)$ amplitudes with the same $\mathbf{n}$ in the coherent state representation (88), implies the asymptotic formula for the vertex amplitude to be given by the unbalanced square of the above formula [167], namely

$$
\begin{aligned}
& A_{v}^{e p r l} \sim \frac{1}{\lambda^{12}}\left[N_{+} e^{i \frac{(1-\gamma)}{2} \sum_{a<b} \lambda j_{a b} \Theta_{a b}^{E}}\right. \\
& \left.+N_{-} e^{-i \frac{(1-\gamma)}{2} \sum_{a<b} \lambda j_{a b} \Theta_{a b}^{E}}\right] \\
& \times\left[N_{+} e^{i \frac{(1+\gamma)}{2} \sum_{a<b} \lambda j_{a b} \Theta_{a b}^{E}}\right. \\
& \left.+N_{-} e^{-i \frac{(1+\gamma)}{2} \sum_{a<b} \lambda j_{a b} \Theta_{a b}^{E}}\right] .
\end{aligned}
$$

One can write the previous expression as

$$
\begin{aligned}
& A_{v}^{e p r l} \sim \frac{1}{\lambda^{12}}\left[2 N_{+} N_{-} \cos \left(S_{\text {Regge }}^{E}\right)\right. \\
& \left.+N_{+}^{2} e^{i \frac{1}{\gamma} S_{R e g g e}^{E}}+N_{-}^{2} e^{-i \frac{1}{\gamma} S_{R e g g e}^{E}}\right] .
\end{aligned}
$$

where

$$
S_{\text {Regge }}^{E}=\sum_{a<b} \lambda \gamma j_{a b} \Theta_{a b}^{E}
$$

is the Regge-like action for $\lambda \gamma j_{a b}=A_{a b}$, the ten triangle areas (according to the LQG area spectrum $[1,2])$. Remarkably, the above asymptotic formula is also valid for the case $\gamma>1$ [80]. The first term in the vertex asymptotics is in essence the expected one: It is the analog of the $6 j$ symbol asymptotics in three-dimensional spin foams. Due to their explicit dependence on the Immirzi parameter, the last two terms are somewhat strange from the theoretical point of view of the continuum field. However, this seems to be a peculiarity of the Riemannian theory alone, as shown by the results of Ref. [166] for the Lorentzian models. Non-geometric configurations are exponentially suppressed 
Papers in Physics, vol. 4, Art. 040004 (2012) / A. Perez

\section{d. Lorentzian EPRL model}

To each solution, one can associate a second solution corresponding to a parity related 4-simplex and, consequently, the asymptotic formula has two terms. It is given, up to a global sign, by the expression

$$
\begin{aligned}
& A_{v}^{e p r l} \sim \frac{1}{\lambda^{12}}\left[N_{+} \exp \left(i \lambda \gamma \sum_{a<b} j_{a b} \Theta_{a b}^{L}\right)\right. \\
& \left.+N_{-} \exp \left(-i \lambda \gamma \sum_{a<b} j_{a b} \Theta_{a b}^{L}\right)\right]
\end{aligned}
$$

where $N_{ \pm}$are constants that do not scale. Nongeometric configurations are exponentially suppressed

In Ref. [171], Freidel and Conrady gave a detailed description of the coherent state representation of the various spin foam models described so far. In particular, they provided the definition of the effective discrete actions associated to each case which we presented in (101). This provides the basic elements for setting up the asymptotic analysis presented in Ref. [170] (the first results of the semiclassical limit of the new spin foam models). This is similar to the studies of the asymptotic of the vertex amplitude reviewed above, but more general in the sense that the semiclassical limit of a full spin foam configuration (involving many vertices) is studied. The result is technically more complex as one studies now critical points of the action associated to a colored complex which, in addition of depending on group variables $g$, depends on the coherent state parameters $\mathbf{n}$. The authors of Ref. [170] write Eq. (101) in the following way:

$$
Z_{f k}^{\gamma}=\sum_{j_{f}} \prod_{f \in \Delta^{\star}} \mathrm{d}_{(1-\gamma) \frac{j_{f}}{2}} \mathrm{~d}_{(1+\gamma) \frac{j_{f}}{2}} W_{\Delta^{\star}}^{\gamma}\left(j_{f}\right),
$$

where

$$
\begin{aligned}
& W_{\Delta^{\star}}^{\gamma}\left(j_{f}\right) \\
& =\int \prod_{e \in \Delta^{\star}} \mathrm{d}_{|1-\gamma| \frac{j_{e f}}{2}} \mathrm{~d}_{(1+\gamma) \frac{j_{e f}}{2}} d n_{e f} d g_{e f}^{-} d g_{e f}^{+} \\
& \times \exp \left(S_{j^{ \pm}, \mathbf{n}}^{f k}\left[g^{ \pm}\right]\right) .
\end{aligned}
$$

They show that those solutions of the equations of motion of the effective discrete action that are nongeometric (i.e., the contrary of Regge-like) are not critical and hence exponentially suppressed in the scaling $j_{f} \rightarrow \lambda j_{f}$ with $\lambda>>1$. If configurations are geometric (i.e., Regge-like), one has two kinds of contributions to the amplitude asymptotics: Those coming from degenerate and non-degenerate configurations. If one (by hand) restricts to the nondegenerate configurations, then one has

$$
\begin{aligned}
& W_{\Delta^{\star}}^{\gamma}\left(j_{f}\right) \sim \frac{c}{\lambda^{\left(33 n_{e}-6 n_{v}-4 n_{f}\right)}} \\
& \times \exp \left(i \lambda S_{\text {Regge }}^{E}\left(\Delta^{\star}, j_{f}\right)\right),
\end{aligned}
$$

where $n_{e}, n_{v}$, and $n_{f}$ denote the number of edges, vertices, and faces in the two complex $\Delta^{\star}$, respectively. There are recent works by M. Han in which asymptotics of general simplicial geometry amplitudes are studied in the context of the EPRL model $[174,175]$.

The problem of computing the two-point function and higher correlation functions in the context of spin foam has received a lot of attention recently. The framework for the definition of the correlation functions in the background independent setting has been generally discussed by Rovelli in Ref. [176], and correspods to a special application of a more general proposal investigated by Oeckl [177-184]. It was then applied to the Barrett-Crane model in Refs. [185-187], where it was discovered that certain components of the twopoint function could not yield the expected result compatible with Regge gravity in the semiclassical limit. This was used as the main motivation for the weakening of the imposition of the Plebanski constraints, leading to the new models. Soon thereafter, it was argued that the difficulties of the Barrett-Crane model where indeed absent in the EPRL model [188]. The two-point function for the EPRL model was calculated in Ref. [189] and it was shown to produce a result in agreement with that of Regge calculus [190,191], in the limit $\gamma \rightarrow 0$.

The fact that, for the new model, the double scaling limit $\gamma \rightarrow 0$ and $j \rightarrow \infty$ with $\gamma j=$ constant defines the appropriate regime where the fluctuation behave as in Regge gravity (in the leading order) has been further clarified in Ref. [192]. This indicates that the quantum fluctuations in the new models are more general than simply metric fluc- 
Papers in Physics, vol. 4, Art. 040004 (2012) / A. Perez

tuations. The fact that the new models are not metric at all scales should not be surprising as we know that the Plebanski constraints that produce metric general relativity out of BF theory have been implemented only semiclassically (in the large spin limit). At the deep Planckian regime, fluctuations are more general than metric. However, it is not clear at this stage why this is controlled by the Immirzi parameter.

All the previous calculations involve a complex with a single four-simplex. The first computation involving more than one simplex was performed in Refs. [187, 193], for the case of the Barrett-Crane model. Certain peculiar properties were found and it is not clear at this stage whether these issues remain in the EPRL model. Higher order correlation functions have been computed in Ref. [194], the results are in agreement with Regge gravity in the $\gamma \rightarrow 0$ limit.

\section{Acknowledgements}

I would like to thank the help from many people in the field that have helped me in various ways. I am grateful to Eugenio Bianchi, Carlo Rovelli and Simone Speziale for the many discussions on aspects and details of the recent literature. Many detailed calculations that contributed to the presentation of the new models in this review were done in collaboration with Mercedes Velázquez to whom I would like to express my gratitude. I would also like to thank You Ding, Florian Conrady, Laurent Freidel, Muxin Han, Merced Montesinos for their help and valuable interaction.

[1] C Rovelli, Quantum Gravity, Cambridge University Press, Cambridge (UK) (2004), Pag. 480 .

[2] T Thiemann, Modern canonical quantum general relativity, Cambridge University Press, Cambridge (UK) (2007), Pag. 819.

[3] A Ashtekar, J Lewandowski, Background independent quantum gravity: A status report, Class. Quant. Grav. 21, R53 (2004).

[4] J Engle, R Pereira, C Rovelli, The loopquantum-gravity vertex-amplitude, Phys. Rev. Lett. 99, 161301 (2007).
[5] J Engle, E Livine, R Pereira, C Rovelli, $L Q G$ vertex with finite Immirzi parameter, Nucl. Phys. B 799, 136 (2008).

[6] L Freidel, K Krasnov, A new spin foam model for 4 d gravity, Class. Quant. Grav. 25, 125018 (2008).

[7] C Rovelli, Zakopane lectures on loop gravity, arXiv:1102.3660 (2011).

[8] A Perez, The spin foam approach to quantum gravity, Liv. Rev. Rel. (in press).

[9] J C Baez, An introduction to spin foam models of quantum gravity and bf theory, Lect. Notes Phys. 543, 25 (2000).

[10] R Oeckl, Discrete gauge theory: From lattices to TQFT, Imperial College Press, London (UK) (2005), Pag. 202.

[11] R Oeckl, H Pfeiffer, The dual of pure nonAbelian lattice gauge theory as a spin foam model, Nucl. Phys. B 598, 400 (2001).

[12] F Girelli, R Oeckl, A Perez, Spin foam diagrammatics and topological invariance, Class. Quant. Grav. 19, 1093 (2002).

[13] D Yetter L Crane, A Categorical construction of 4-D topological quantum field theories, In: Quantum Topology, Eds. L Kaufmann, R Baadhio, Pag. 120, World Scientific, Singapore (1993).

[14] D N Yetter, L Crane, L Kauffman, State-sum invariants of 4-manifolds, J. Knot Theor. Ramif. 6, 177 (1997).

[15] J C Baez, A Perez, Quantization of strings and branes coupled to BF theory, Adv. Theor. Math. Phys. 11, 3 (2007).

[16] W J Fairbairn, A Perez, Extended matter coupled to BF theory, Phys. Rev. D, 78, 024013 (2008).

[17] M Montesinos, A Perez, Two-dimensional topological field theories coupled to fourdimensional BF theory, Phys. Rev. D 77, 104020 (2008).

[18] G 't Hooft, A locally finite model for gravity, Found. Phys. 38, 733 (2008). 
Papers in Physics, vol. 4, ART. 040004 (2012) / A. Perez

[19] L Freidel, J Kowalski-Glikman, A Starodubtsev, Particles as Wilson lines of gravitational field, Phys. Rev. D 74, 084002 (2006).

[20] E R Livine, A Perez, C Rovelli, 2D manifoldindependent spinfoam theory, Class. Quant. Grav. 20, 4425 (2003).

[21] R Jackiw, Liouville field theory: A twodimensional model for gravity? In: Quantum theory of gravity, Eds. S M Christensen, B S DeWitt, Pag. 403, Adam Hilger Ltd., Bristol (1984).

[22] C Teitelboim, The Hamiltonian structure of two-dimensional space-time and its relation with the conformal anomaly, In: Quantum theory of gravity, Eds. S M Christensen, B S DeWitt, Pag. 403, Adam Hilger Ltd., Bristol (1984).

[23] C P Constantinidis, O Piguet, A Perez, Quantization of the Jackiw-Teitelboim model, Phys. Rev. D 79, 084007 (2009).

[24] D Oriti, C Rovelli, S Speziale, Spinfoam 2d quantum gravity and discrete bundles, Class. Quant. Grav. 22, 85 (2005).

[25] S Carlip, Quantum gravity in 2+1 dimensions, Cambridge University Press, Cambridge (UK) (1998), Pag. 276.

[26] T Regge, G Ponzano, Semiclassical limit of Racah coeficients, In: Spectroscopy and group theoretical methods in physics, Eds. F Block et al., North-Holland, Amsterdam (1968).

[27] J W Barrett, I Naish-Guzman, The PonzanoRegge model, Class. Quant. Grav. 26, 155014 (2009).

[28] K Noui, A Perez, Three dimensional loop quantum gravity: Physical scalar product and spin foam models, Class. Quant. Grav. 22, 1739 (2005).

[29] L Freidel, D Louapre, Diffeomorphisms and spin foam models, Nucl. Phys. B 662, 279 (2003).
[30] V Bonzom, M Smerlak, Bubble divergences from cellular cohomology, Lett. Math. Phys. 93, 295 (2010).

[31] V Bonzom, M Smerlak, Bubble divergences from twisted cohomology, arXiv:1008.1476 (2010).

[32] V Bonzom, M Smerlak, Bubble divergences: sorting out topology from cell structure, Ann. Henri Poincare 13, 185 (2012).

[33] O Y Viro, V G Turaev, Statesum invariants of 3-manifolds and quantum 6j-symbols, Topology 31, 865 (1992).

[34] J W Barrett, J M Garcia-Islas, J F Martins, Observables in the Turaev-Viro and CraneYetter models, J. Math. Phys. 48, 093508 (2007).

[35] K Noui, A Perez, Observability and geometry in three dimensional quantum gravity, In: Quantum theory and symmetries, Eds. P C Argyres et al., Pag. 641, World Scientific, Singapore (2004).

[36] K Noui, A Perez, Three dimensional loop quantum gravity: Coupling to point particles, Class. Quant. Grav. 22, 4489 (2005).

[37] L Freidel, D Louapre, Ponzano-Regge model revisited. I: Gauge fixing, observables and interacting spinning particles, Class. Quant. Grav. 21, 5685 (2004).

[38] L Freidel, E R Livine, Ponzano-Regge model revisited. III: Feynman diagrams and effective field theory, Class. Quant. Grav. 23, 2021 (2006).

[39] L Freidel, E R Livine, Effective 3d quantum gravity and non-commutative quantum field theory, Phys. Rev. Lett. 96, 221301 (2006).

[40] W J Fairbairn, Fermions in threedimensional spinfoam quantum gravity, Gen. Rel. Grav. 39, 427 (2007).

[41] R J Dowdall, W J Fairbairn, Observables in 3d spinfoam quantum gravity with fermions, Gen. Rel. Grav. 43, 1263 (2011).

[42] S Speziale, Coupling gauge theory to spinfoam 3d quantum gravity, Class. Quant. Grav. 24, 5139 (2007). 
Papers in Physics, vol. 4, ART. 040004 (2012) / A. Perez

[43] W J Fairbairn, E R Livine, 3d spinfoam quantum gravity: Matter as a phase of the group field theory, Class. Quant. Grav. 24, 5277 (2007).

[44] E R Livine, R Oeckl, Three-dimensional quantum supergravity and supersymmetric spin foam models, Adv. Theor. Math. Phys. 7, 951 (2004).

[45] V Baccetti, E R Livine, J P Ryan, The particle interpretation of $N=1$ supersymmetric spin foams, Class. Quant. Grav. 27, 225022 (2010).

[46] V Bonzom, E R Livine, Yet another recursion relation for the 6j-symbol, arXiv:1103.3415 (2011).

[47] M Dupuis, E R Livine, The 6j-symbol: Recursion, correlations and asymptotics, Class. Quant. Grav. 27, 135003 (2010).

[48] V Bonzom, E R Livine, S Speziale, Recurrence relations for spin foam vertices, Class. Quant. Grav. 27, 125002 (2010).

[49] E R Livine, S Speziale, A new spinfoam vertex for quantum gravity, Phys. Rev. D 76, 084028 (2007).

[50] T Thiemann, Coherent states on graphs, Prepared for 9th Marcel Grossmann Meeting on Recent Developments in Theoretical and Experimental General Relativity, Gravitation and Relativistic Field Theories (MG 9), Rome (Italy), 2-9 July (2000).

[51] T Thiemann, Gauge field theory coherent states (gcs). i: General properties, Class. Quant. Grav. 18, 2025 (2001).

[52] H Sahlmann, T Thiemann, O Winkler, Coherent states for canonical quantum general relativity and the infinite tensor product extension, Nucl. Phys. B 606, 401 (2001).

[53] T Thiemann, O Winkler, Gauge field theory coherent states (GCS) 2. Peakedness properties, Class. Quant. Grav. 18, 2561 (2001).

[54] T Thiemann, O Winkler, Gauge field theory coherent states (GCS) 3. Ehrenfest theorems, Class. Quant. Grav. 18, 4629 (2001).
[55] T Thiemann, O Winkler, Gauge field theory coherent states (GCS) 4. Infinite tensor product and thermodynamical limit, Class. Quant. Grav. 18, 4997 (2001).

[56] T Thiemann, Complexifier coherent states for quantum general relativity, Class. Quant. Grav. 23, 2063 (2006).

[57] B Bahr, T Thiemann, Gauge-invariant coherent states for Loop Quantum Gravity. I. Abelian gauge groups, Class. Quant. Grav. 26, 045011 (2009).

[58] B Bahr, T Thiemann, Gauge-invariant coherent states for loop quantum gravity. II. Non-Abelian gauge groups, Class. Quant. Grav. 26, 045012 (2009).

[59] C Flori, T Thiemann, Semiclassical analysis of the Loop Quantum Gravity volume operator. I. Flux Coherent States, arXiv:0812.1537 (2008).

[60] E Bianchi, E Magliaro, C Perini, Coherent spin-networks, Phys. Rev. D 82, 024012 (2010).

[61] F Conrady, L Freidel, Quantum geometry from phase space reduction, J. Math. Phys. 50, 123510 (2009).

[62] E Buffenoir, P Roche, Harmonic analysis on the quantum Lorentz group, Commun. Math. Phys. 207, 499 (1999).

[63] W Ruhl, The Lorentz group and harmonic analysis, W. A. Benjamin Inc., New York (1970).

[64] I M Gelfand, Generalized Functions, Academic Press, New York (1966), Vol. 5.

[65] I M Gelfand, R A Minlos, Z Ya Shapiro, Representations of the rotation and Lorentz groups and their applications, Pergamon Press, Oxford (1963).

[66] J W Barrett, L Crane, Relativistic spin networks and quantum gravity, J. Math. Phys. 39, 3296 (1998).

[67] Y Ding, C Rovelli, The volume operator in covariant quantum gravity, Class. Quant. Grav. 27, 165003 (2010). 
[68] Y Ding, M Han, C Rovelli, Generalized Spinfoams, Phys. Rev. D 83, 124020 (2011).

[69] S Alexandrov, The new vertices and canonical quantization, Phys. Rev. D 82, 024024 (2010).

[70] C Rovelli, S Speziale, Lorentz covariance of loop quantum gravity, Phys. Rev. D 83, 104029 (2011).

[71] W M Wieland, Twistorial phase space for complex Ashtekar variables, Class. Quant. Grav. 29, 045007 (2012)..

[72] M Dupuis, L Freidel, E R Livine, S Speziale, Holomorphic Lorentzian simplicity constraints, arXiv:1107.5274 (2011).

[73] E R Livine, S Speziale, J Tambornino, Twistor Networks and Covariant Twisted Geometries, Phys. Rev. D 85, 064002 (2012).

[74] J C Baez, Spin foam models, Class. Quant. Grav. 15, 1827 (1998).

[75] M Bojowald, A Perez, Spin foam quantization and anomalies, Gen. Rel. Grav. 42, 877 (2010).

[76] B Bahr, F Hellmann, W Kaminski, M Kisielowski, J Lewandowski, Operator spin foam models, Class. Quant. Grav. 28, 105003 (2011).

[77] W Kaminski, M Kisielowski, J Lewandowski, The EPRL intertwiners and corrected partition function, Class. Quant. Grav. 27, 165020 (2010).

[78] E Alesci, E Bianchi, E Magliaro, C Perini, Asymptotics of LQG fusion coefficients, Class. Quant. Grav. 27, 095016 (2010).

[79] E R. Livine, S Speziale, Consistently solving the simplicity constraints for spinfoam quantum gravity, Europhys. Lett. 81, 50004 (2008).

[80] J W Barrett, R J Dowdall, W J Fairbairn, H Gomes, F Hellmann, Asymptotic analysis of the EPRL four-simplex amplitude, J. Math. Phys. 50, 112504 (2009).
[81] S Alexandrov, Simplicity and closure constraints in spin foam models of gravity, Phys. Rev. D 78, 044033 (2008).

[82] S Alexandrov, Spin foam model from canonical quantization, Phys. Rev. D 77, 024009 (2008).

[83] V Bonzom, Spin foam models for quantum gravity from lattice path integrals, Phys. Rev. D 80, 064028 (2009).

[84] V Bonzom, From lattice BF gauge theory to area-angle Regge calculus, Class. Quant. Grav. 26, 155020 (2009).

[85] V Bonzom, E R Livine, A Lagrangian approach to the Barrett-Crane spin foam model, Phys. Rev. D 79, 064034 (2009).

[86] M Han, T Thiemann, Commuting simplicity and closure constraints for $4 D$ spin foam models, arXiv:1010.5444 (2010).

[87] A Baratin, C Flori, T Thiemann, The Holst spin foam model via cubulations, arXiv:0812.4055 (2008).

[88] M Dupuis, E R Livine, Revisiting the simplicity constraints and coherent intertwiners, Class. Quant. Grav. 28, 085001 (2011).

[89] L Freidel, E R Livine, U(N) Coherent States for Loop Quantum Gravity, J. Math. Phys. 52, 052502 (2011).

[90] L Freidel, E R Livine, The fine structure of $S U(2)$ intertwiners from $U(N)$ representations, J. Math. Phys. 51, 082502 (2010).

[91] E F Borja, L Freidel, I Garay, E R Livine, $U(N)$ tools for loop quantum gravity: The return of the spinor, Class. Quant. Grav. 28, 055005 (2011).

[92] E R Livine, J Tambornino, Spinor representation for loop quantum gravity, J. Math. Phys. 53, 012503 (2012).

[93] B Dittrich, J P Ryan, Simplicity in simplicial phase space, Phys. Rev. D 82, 064026 (2010).

[94] J Engle, R Pereira, Regularization and finiteness of the Lorentzian LQG vertices, Phys. Rev. D 79, 084034 (2009). 
Papers in Physics, vol. 4, Art. 040004 (2012) / A. Perez

[95] L Liu, M Montesinos, A Perez, A topological limit of gravity admitting an $S U(2)$ connection formulation, Phys. Rev. D 81, 064033 (2010).

[96] Y Ding, C Rovelli, Physical boundary Hilbert space and volume operator in the Lorentzian new spin-foam theory, Class. Quant. Grav. 27, 205003 (2010).

[97] W Kaminski, J Lewandowski, T Pawlowski, Quantum constraints, Dirac observables and evolution: group averaging versus Schroedinger picture in $L Q C$, Class. Quant. Grav. 26, 245016 (2009).

[98] W Kaminski, M Kisielowski, J Lewandowski, Spin-foams for all loop quantum gravity, Class. Quant. Grav. 27, 095006 (2010).

[99] L Freidel, E R Livine, Spin networks for noncompact groups, J. Math. Phys. 44, 1322 (2003).

[100] S Alexandrov, E R Livine, SU(2) loop quantum gravity seen from covariant theory, Phys. Rev. D 67, 044009 (2003).

[101] E R Livine, Projected spin networks for Lorentz connection: Linking spin foams and loop gravity, Class. Quant. Grav. 19, 5525 (2002).

[102] S Alexandrov, E Buffenoir, P Roche, Plebanski theory and covariant canonical formulation, Class. Quant. Grav. 24, 2809 (2007).

[103] S Alexandrov, Reality conditions for Ashtekar gravity from Lorentz- covariant formulation, Class. Quant. Grav. 23, 1837 (2006).

[104] S Alexandrov, Hilbert space structure of covariant loop quantum gravity, Phys. Rev. D 66, 024028 (2002).

[105] S Alexandrov, Choice of connection in loop quantum gravity, Phys. Rev. D 65, 024011 (2002).

[106] S Alexandrov, SO $(4, C)$-covariant AshtekarBarbero gravity and the Immirzi parameter, Class. Quant. Grav. 17, 4255 (2000).
[107] S Alexandrov, I Grigentch, D Vassilevich, $S U$ (2)-invariant reduction of the $3+1$ dimensional Ashtekar's gravity, Class. Quant. Grav. 15, 573 (1998).

[108] M Dupuis, E R Livine, Lifting SU(2) spin networks to projected spin networks, Phys. Rev. D 82, 064044 (2010).

[109] B Bahr, On knottings in the physical Hilbert space of $L Q G$ as given by the EPRL model, Class. Quant. Grav. 28, 045002 (2011).

[110] E Buffenoir, M Henneaux, K Noui, Ph Roche, Hamiltonian analysis of Plebanski theory, Class. Quant. Grav. 21, 5203 (2004).

[111] J Engle, M Han, T Thiemann, Canonical path integral measures for Holst and Plebanski gravity. I. Reduced Phase Space Derivation, Class. Quant. Grav. 27, 245014 (2010).

[112] M Han, Canonical path-integral measures for Holst and Plebanski gravity. II. Gauge invariance and physical inner product, Class. Quant. Grav. 27, 245015 (2010).

[113] E Bianchi, D Regoli, C Rovelli, Face amplitude of spinfoam quantum gravity, Class. Quant. Grav. 27, 185009 (2010).

[114] K Noui, A Perez, Three dimensional loop quantum gravity: Physical scalar product and spin foam models, Class. Quant. Grav. 22, 1739 (2005).

[115] V Bonzom, L Freidel, The Hamiltonian constraint in 3d Riemannian loop quantum gravity, Class. Quant. Grav. 28, 195006 (2011).

[116] V Bonzom, A taste of Hamiltonian constraint in spin foam models, arXiv:1101.1615 (2011).

[117] B Dittrich, S Speziale, Area-angle variables for general relativity, New J. Phys. 10, 083006 (2008).

[118] E Alesci, K Noui, F Sardelli, Spin-foam models and the physical scalar product, Phys. Rev. D 78, 104009 (2008).

[119] E R Livine, D Oriti, J P Ryan, Effective Hamiltonian constraint from group field theory, Class. Quant. Grav. 28, 245010 (2011). 
Papers in Physics, vol. 4, Art. 040004 (2012) / A. Perez

[120] M Han, T Thiemann, On the relation between operator constraint, master constraint, reduced phase space, and path integral quantisation, Class. Quant. Grav. 27, 225019 (2010).

[121] M Han, T Thiemann, On the relation between rigging inner product and master constraint direct integral decomposition, J. Math. Phys. 51, 092501 (2010).

[122] M Han, A path-integral for the master constraint of loop quantum gravity, Class. Quant. Grav. 27, 215009 (2010).

[123] B Dittrich, P A Hohn, From covariant to canonical formulations of discrete gravity, Class. Quant. Grav. 27, 155001 (2010).

[124] F Conrady, J Hnybida, Unitary irreducible representations of $S L(2, C)$ in discrete and continuous $S U(1,1)$ bases, J. Math. Phys. 52, 012501 (2011).

[125] F Conrady, Spin foams with timelike surfaces, Class. Quant. Grav. 27, 155014 (2010).

[126] F Conrady, J Hnybida, A spin foam model for general Lorentzian 4-geometries, Class. Quant. Grav. 27, 185011 (2010).

[127] A Perez, C Rovelli, 3+1 spinfoam model of quantum gravity with spacelike and timelike components, Phys. Rev. D 64, 064002 (2001).

[128] D Oriti, H Pfeiffer, A spin foam model for pure gauge theory coupled to quantum gravity, Phys. Rev. D 66, 124010 (2002).

[129] M Han, C Rovelli, Spinfoam fermions: PCT symmetry, Dirac determinant, and correlation functions, arXiv:1101.3264 (2011).

[130] E Bianchi et al., Spinfoam fermions, arXiv:1012.4719 (2010).

[131] S Alexander, A Marciano, R A Tacchi, Towards a Spin-foam unification of gravity, Yang-Mills interactions and matter fields, arXiv:1105.3480 (2011).

[132] J W Barrett, L Crane, A lorentzian signature model for quantum general relativity, Class. Quant. Grav. 17, 3101 (2000).
[133] K Noui, P Roche, Cosmological deformation of Lorentzian spin foam models, Class. Quant. Grav. 20, 3175 (2003).

[134] Y Ding, M Han, On the asymptotics of quantum group spinfoam model, arXiv:1103.1597 (2011).

[135] M Han, 4-dimensional spin-foam model with quantum Lorentz group, J. Math. Phys. 52, 072501 (2011).

[136] W J Fairbairn, C Meusburger, Quantum deformation of two four-dimensional spin foam models, J. Math. Phys. 53, 022501 (2012).

[137] M Han, Cosmological constant in LQG vertex amplitude, arXiv:1105.2212 (2011).

[138] E Bianchi, T Krajewski, C Rovelli, F Vidotto, Cosmological constant in spinfoam cosmology, Phys. Rev. D 83, 104015 (2011).

[139] F Vidotto, Spinfoam Cosmology: quantum cosmology from the full theory, arXiv:1011.4705 (2010).

[140] A Henderson, C Rovelli, F Vidotto, E Wilson-Ewing, Local spinfoam expansion in loop quantum cosmology, Class. Quant. Grav. 28, 025003 (2011).

[141] E Bianchi, C Rovelli, F Vidotto, Towards spinfoam cosmology, Phys. Rev. D 82, 084035 (2010).

[142] C Rovelli, F Vidotto, On the spinfoam expansion in cosmology, Class. Quant. Grav. 27, 145005 (2010).

[143] C Rovelli, F Vidotto, Stepping out of Homogeneity in Loop Quantum Cosmology, Class. Quant. Grav. 25, 225024 (2008).

[144] M Bojowald, Loop quantum cosmology, Liv. Rev. Rel. 8, 11 (2005).

[145] A Ashtekar, M Campiglia, A Henderson, Path integrals and the WKB approximation in loop quantum cosmology, Phys. Rev. D 82, 124043 (2010).

[146] A Ashtekar, M Campiglia, A Henderson, Casting loop quantum cosmology in the spin foam paradigm, Class. Quant. Grav. 27, 135020 (2010). 
[147] A Ashtekar, Ml Campiglia, A Henderson, Loop quantum cosmology and spin foams, Phys. Lett. B 681, 347 (2009).

[148] M Campiglia, A Henderson, W Nelson, Vertex expansion for the Bianchi I model, Phys. Rev. D 82, 064036 (2010).

[149] K Krasnov, Renormalizable non-metric quantum gravity? arXiv:hep-th/0611182 (2006).

[150] K Krasnov, On deformations of Ashtekar's constraint algebra, Phys. Rev. Lett. 100, 081102 (2008).

[151] K Krasnov, Plebanski gravity without the simplicity constraints, Class. Quant. Grav. 26, 055002 (2009).

[152] K Krasnov, Gravity as BF theory plus potential, Int. J. Mod. Phys. A 24, 2776 (2009).

[153] K Krasnov, Metric Lagrangians with two propagating degrees of freedom, Europhys. Lett. 89, 30002 (2010).

[154] S Speziale, Bi-metric theory of gravity from the non-chiral Plebanski action, Phys. Rev. D 82, 064003 (2010).

[155] M P Reisenberger, C Rovelli, Spacetime as a feynman diagram: the connection formulation, Class. Quant. Grav. 18, 121 (2001).

[156] M P Reisenberger, C Rovelli, Spin foams as feynman diagrams, In: 2001, a relativistic spacetime odyssey. Eds. I Ciufolini, D Dominici, L Lusanna, Pag. 431, World Scientific, Singapore (2003).

[157] J Magnen, K Noui, V Rivasseau, M Smerlak, Scaling behaviour of three-dimensional group field theory, Class. Quant. Grav. 26, 185012 (2009).

[158] L Freidel, D Louapre, Non-perturbative summation over $3 D$ discrete topologies, Phys. Rev. D 68, 104004 (2003).

[159] R Gurau, Colored Group Field Theory, Commun. Math. Phys. 304, 69 (2011).

[160] R Gurau, The 1/N expansion of colored tensor models, Ann. Henri Poincare 12, 829 (2011).
[161] R Gurau, A generalization of the Virasoro algebra to arbitrary dimensions, Nucl. Phys. B 852, 592 (2011).

[162] V Bonzom, R Gurau, A Riello, V Rivasseau, Critical behavior of colored tensor models in the large $N$ limit, Nucl. Phys. B 853, 174 (2011).

[163] R Gurau, The complete $1 / N$ expansion of colored tensor models in arbitrary dimension, Ann. Henri Poincare 13, 399 (2011).

[164] R Gurau, V Rivasseau, The $1 / N$ expansion of colored tensor models in arbitrary dimension, Europhys. Lett 95, 50004 (2011).

[165] J P Ryan, Tensor models and embedded Riemann surfaces, Phys. Rev. D 85, 024010 (2012).

[166] J W Barrett, R J Dowdall, W J Fairbairn, F Hellmann, R Pereira, Lorentzian spin foam amplitudes: graphical calculus and asymptotics, Class. Quant. Grav. 27, 165009 (2010).

[167] J W Barrett, R J Dowdall, W J Fairbairn, H Gomes, F Hellmann, A Summary of the asymptotic analysis for the EPRL amplitude, In: AIP Conf. Proc. 1196, Pag. 36, (2009).

[168] J W Barrett, W J Fairbairn, F Hellmann, Quantum gravity asymptotics from the $S U(2)$ $15 j$ symbol, Int. J. Mod. Phys. A 25, 2897 (2010).

[169] J W Barrett et al., Asymptotics of 4d spin foam models, Gen. Relat. Gravit. 43, 2421 (2011).

[170] F Conrady, L Freidel, On the semiclassical limit of $4 d$ spin foam models, Phys. Rev. D 78, 104023 (2008).

[171] F Conrady, L Freidel, Path integral representation of spin foam models of $4 d$ gravity, Class. Quant. Grav. 25, 245010 (2008).

[172] J W Barrett, Ch M Steele, Asymptotics of relativistic spin networks, Class. Quant. Grav. 20, 1341 (2003). 
Papers in Physics, vol. 4, ART. 040004 (2012) / A. Perez

[173] J W Barrett, R M Williams, The asymptotics of an amplitude for the 4-simplex, Adv. Theor. Math. Phys. 3, 209 (1999).

[174] M Han, M Zhang, Asymptotics of spinfoam amplitude on simplicial manifold: Euclidean theory, arXiv:1109.0500 (2011).

[175] M Han, M Zhang, Asymptotics of spinfoam amplitude on simplicial manifold: Lorentzian theory, arXiv:1109.0499 (2011).

[176] C Rovelli, Graviton propagator from background-independent quantum gravity, Phys. Rev. Lett. 97, 151301 (2006).

[177] R Oeckl, Affine holomorphic quantization, arXiv:1104.5527 (2011).

[178] R Oeckl, Observables in the general boundary formulation, In: Quantum field theory and gravity, Eds. F Finster et al., Pag. 137, Birkhäuser, Basel, (2012).

[179] R Oeckl, Holomorphic quantization of linear field theory in the general boundary formulation, arXiv:1009.5615 (2010).

[180] D Colosi, Robert Oeckl, On unitary evolution in quantum field theory in curved spacetime, Open Nucl. Part. Phys. J. 4, 13 (2011).

[181] D Colosi, Robert Oeckl, States and amplitudes for finite regions in a two-dimensional Euclidean quantum field theory, J. Geom. Phys. 59, 764 (2009).

[182] D Colosi, R Oeckl, Spatially asymptotic Smatrix from general boundary formulation, Phys. Rev. D 78, 025020 (2008).

[183] D Colosi, R Oeckl, S-matrix at spatial infinity, Phys. Lett. B 665, 310 (2008).
[184] R Oeckl, Probabilites in the general boundary formulation, J. Phys. Conf. Ser. 67, 012049 (2007).

[185] E Alesci, C Rovelli, The complete LQG propagator. II. Asymptotic behavior of the vertex, Phys. Rev. D 77, 044024 (2008).

[186] E Alesci, C Rovelli, The complete LQG propagator. I. Difficulties with the Barrett-Crane vertex, Phys. Rev. D 76, 104012 (2007).

[187] E Bianchi, L Modesto, C Rovelli, S Speziale, Graviton propagator in loop quantum gravity, Class. Quant. Grav. 23, 6989 (2006).

[188] E Alesci, E Bianchi, C Rovelli, LQG propagator: III. The new vertex, Class. Quant. Grav. 26, 215001 (2009).

[189] E Bianchi, E Magliaro, C Perini, LQG propagator from the new spin foams, Nucl. Phys. B 822, 245 (2009).

[190] E Bianchi, A Satz, Semiclassical regime of Regge calculus and spin foams, Nucl. Phys. B 808, 546 (2009).

[191] E Magliaro, C Perini, Comparing LQG with the linearized theory, Int. J. Mod. Phys. A 23, 1200 (2008).

[192] E Magliaro, C Perini, Regge gravity from spinfoams, arXiv:1105.0216 (2011).

[193] D Mamone, C Rovelli, Second-order amplitudes in loop quantum gravity, Class. Quant. Grav. 26, 245013 (2009).

[194] C Rovelli, M Zhang, Euclidean three-point function in loop and perturbative gravity, Class. Quant. Grav. 28, 175010 (2011). 